\title{
Kernos
}

Revue internationale et pluridisciplinaire de religion grecque antique

$10 \mid 1997$

Varia

\section{L'arrhéphorie : initiation ou rite civique? Un cas d'école}

\section{Guy Donnay}

\section{OpenEdition \\ Journals}

Édition électronique

URL : http://journals.openedition.org/kernos/656

DOI : $10.4000 /$ kernos.656

ISSN : 2034-7871

\section{Éditeur}

Centre international d'étude de la religion grecque antique

\section{Édition imprimée}

Date de publication : 1 janvier 1997

Pagination : 177-205

ISSN : 0776-3824

\section{Référence électronique}

Guy Donnay, «L'arrhéphorie : initiation ou rite civique ? Un cas d'école », Kernos [En ligne], 10 | 1997, mis en ligne le 12 avril 2011, consulté le 30 avril 2019. URL : http://journals.openedition.org/ kernos/656 ; DOI : 10.4000/kernos.656 


\title{
L'Arrhéphorie : initiation ou rite civique?
}

\author{
Un cas d'école
}

Il y a dix ans paraissaient, indépendamment l'une de l'autre, deux études sur l'arrhéphorie qui, au terme d'une nouvelle analyse de l'ensemble des sources, aboutissaient à des conclusions diamétralement opposées ${ }^{1}$. Tandis que, dans L'Antiquité classique, Laurence Van Sichelen affirmait qu'il ne pouvait s'agir d'un rite d'initiation, dans son livre La fille d'Atbènes, Pierre Brulé, tout en reconnaissant que « la seule documentation sur les arrhéphores est insuffisante pour laisser la possibilité de trancher sur l'interprétation sociale du rituel $»^{2}$, n'en prenait pas moins position en faveur de l'interprétation initiatique défendue avant lui par $\mathrm{H}$. Jeanmaire et A. Brelich. Cette documentation est-elle donc à ce point indigente que chacun puisse ainsi l'interpréter à sa guise? En fait, jusqu'à présent, la plupart des chercheurs se sont surtout acharnés sur le témoignage de Pausanias, qu'ils ont souvent sollicité au-delà du raisonnable ${ }^{3}$. Ils n'ont guère accordé d'intérêt, en revanche, aux données épigraphiques, qui constituent pourtant des témoins de première main. Quant aux lexiques et scholies, qui sont nos sources principales sur l'arrhéphorie - comme sur la plupart des réalités cultuelles de la Grèce antique -, on s'est généralement contenté d'y puiser des arguments pour ou contre telle ou telle théorie a priori.

J'ai donc estimé nécessaire de reprendre l'ensemble du dossier, en mettant l'accent sur les données qui me paraissaient avoir été négligées par mes prédé-

L. VAN SICHELEN, Nouvelles orientations dans l'étude de l'arrbépborie attique, in AC, 56 (1987), p. 88-102, où sont résumées les conclusions d'un mémoire de licence présenté sous ma direction à I’Université de Bruxelles en 1982. P. BRuLÉ, La fille d'Athènes, La religion des filles à Athènes à l'époque classique. Mytbes, cultes et société, Paris, 1987, chap. 1.2.1. L'arrbépborte, p. 79-98; le livre est une version remaniée d'une thèse soutenue en 1985 à la Faculté des Lettres de Besançon sous la direction de P. Lévêque.

2

p. cit. (n. 1), p. 87

3 Depuis l'article de J.E. HARRISON, On the meaning of the term Arrhephoroi, in $C R, 3$ (1889), p. 187, on retiendra surtout, outre les deux études déjà citées, H. JEANMAIRE, Couroi et courètes, Paris, 1939, p. 264-268; F.R. ADRADOS, Sobre los Arreforias o Erreforias, in Emerita, 19 (1951), p. 117-133; W. BURKERT, Kekropidensage und Arbepboria. Vom Initiationsritus zum Panathenäenfest, in Hermes, 94 (1966), p. 1-25; A. BRELICH, Paides e partbenoi, Rome, 1969, p. 231-238 et 267-273; Cl. BÉRARD, ANODOI. Essai sur l'imagerie des passages chtboniens, Rome, 1974, p. 117-125; N. ROBERTSON, The riddle of the Arrbephoria at Athens, in HSPh, 87 (1983), p. 241-288; V. PIRENNEDELFORGE, L'Apbrodite grecque, Athènes-Liège, 1994 (Kemos, suppl. 4), p. 48-59. 
cesseurs. Pour ce faire, j'ai tenté de rassembler, dans la mesure du possible, la totalité des sources dont nous disposons sur l'arrhéphorie: on en trouvera la liste en annexe ${ }^{4}$. Afin d'alléger les références, chaque source sera dorénavant désignée par le sigle qui la précède dans cette liste. Celle-ci se veut exhaustive, dans les limites des textes publiés ${ }^{5}$. Les sources y sont classées en quatre catégories - sources littéraires (A), lexiques (L), scholies (S), inscriptions (I) - et, à l'intérieur de chaque catégorie, par ordre chronologique. La classification n'échappe pas à un certain arbitraire: par exemple, le fragment de Dinarque est classé sous sa source lexicographique [L1], tandis que celui d'Élien [A10] et celui attribué abusivement à Platon le Comique [A11] figurent parmi les «auteurs »; le fragment de Tryphon conservé par Athénée [A6] eût été peut-être mieux à sa place parmi les lexiques...

\section{Le vocabulaire de l'arrhéphorie}

Pour commencer, il n'est sans doute pas inutile de préciser le vocabulaire de l'arrhéphorie, dont les fluctuations orthographiques ont intrigué tant les Anciens que les Modernes. Les sources recensées contiennent 76 attestations d'àppnфópos et des termes apparentés, dont les variantes se répartissent comme suit:

Formes en $\alpha^{-}$:

áppnфópos : A4, A5, A6, A8, A9, A10, A11, L1, L3b, L4a, L4b, L5a, L5c, L5f, L6, L7, L9a, L11, I16, I24

áppnфopía : A2, L3a, L4a, L5c, L5d, L8, L9b, L11, S1

áppnтофорía (féminin singulier) : L7

àppฑтофópla (neutre pluriel) : A12, S2

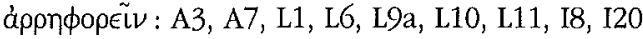

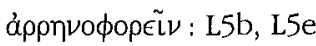

\section{A $\quad$ L $\quad S \quad$ I Total}

$\begin{array}{ccccc}7 & 11 & - & 2 & 20 \\ 1 & 7 & 1 & - & 9 \\ - & 1 & - & - & 1 \\ 1 & - & 1 & - & 2 \\ 2 & 5 & - & 2 & 9 \\ - & 2 & - & - & 2\end{array}$

$\begin{array}{lllll}11 & 26 & 2 & 4 & 43\end{array}$

Formes en $\epsilon^{-6}$ :

єppnфópos : L2, L3c, I21

єрбпфópos : 122, I23

$\begin{array}{lll}-\quad 2 & - & 1 \\ - & - & 2\end{array}$

4 Ci-dessous p. 203-205. J'ai pris pour base le dossier constitué par L. Van Sichelen en 1982 vérifié et complété, notamment à l'aide de l'index du Thesaurus Linguae Graecae de l'Université de Californie. Je me suis limité aux textes, car, dans l'état actuel des recherches, l'iconographie de l'arrhéphorie est pratiquement inexistante (cf. BRULÉ, op. cit. [n. 1], p. 97 sq.).

5 Pour éviter les doubles emplois, je n’ai retenu des lexiques dérivés de l'Etymologicum genuinum (Etymologicum Magnum et Magna grammatica, entre autres) que les notices qui ne figurent pas dans l'archétype ou qui présentent des variantes significatives par rapport à lui.

6 Compte tenu de l'hésitation des manuscrits entre l'esprit doux et l'esprit rude (v. ci-dessous), j'ai préféré ne pas noter d'esprit sur l'e initial. 
єрpпфорі́a : L3a, L4a, L7, L8

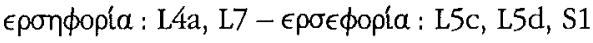

єррпфорєг̃ : I1, I4, I5, I6, I7, I12, I13, I14, I17, I19, I25

$$
\begin{array}{ccccc}
- & 4 & - & - & 4 \\
- & 4 & 1 & - & 5 \\
- & - & - & 11 & 11 \\
\hline- & 10 & 1 & 14 & 25
\end{array}
$$

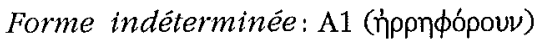

Attestations épigrapbiques dont l'initiale n'est pas conservée:

I3, I11, I15, I18

Restitutions épigraphiques incertaines: $\mathrm{I} 2, \mathrm{I} 9, \mathrm{I} 10$

\begin{tabular}{ll}
4 \\
3 \\
& \\
\hline Total général & 76
\end{tabular}

Ce tableau ne tient compte que des leçons retenues par les éditeurs ${ }^{7}$. En scrutant les apparats critiques des lexiques, on trouve, en outre, çà et là áppnoópı́ accentué proparoxyton, c'est-à-dire au neutre pluriel plutôt qu'au féminin singulier. Les manuscrits de Denys d'Halicarnasse [A5] hésitent entre àppnфópol et àppптофópol. Les formes en $\epsilon$ - ont été particulièrement maltraitées par les copistes, qui les écrivent tantôt avec l'esprit doux, tantôt avec l'esprit rude, tantôt avec $-\rho \rho-$, tantôt avec $-\rho \sigma-$, suivis indifféremment de $\eta$ ou de $\epsilon$, voire de $\iota$ (sans doute pour $\eta$ par iotacisme). L'esprit doux semble néanmoins avoir été la graphie normale; on le trouve même, par contagion, sur l'initiale d' "Epon dans les étymologies fondées sur ce nom (v. ci-dessous). Les inscriptions, qui ne notent pas l'aspiration initiale, ne nous sont d'aucun secours pour trancher en faveur de l'un ou l'autre esprit. En revanche, leur orthographe habituelle est $\epsilon \rho \rho \eta$-; seules

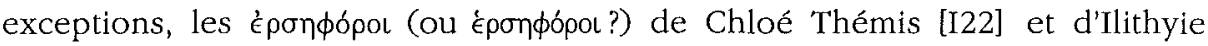
d'Agrai [I23], qui sont tardives. Les formes en $\epsilon \rho \sigma \epsilon-$ de la tradition manuscrite sont donc suspectes.

Majoritaires dans les inscriptions ${ }^{8}$, les formes en $\epsilon$ - sont absentes de la tradition littéraire - ou, du moins, des manuscrits qui nous l'ont transmise ${ }^{9}$. Les lexicographes connaissent les deux, mais privilégient les formes en $\alpha$-: sur seize lemmes, trois seulement adoptent une forme en $\epsilon$ - [L2, L3c, L5d], dont un se contente de renvoyer à un lemme en $\alpha$-. Les Anciens justifiaient l'une et l'autre orthographe à l'aide d'étymologies ad boc, dont on trouve l'écho dans les lexiques. Celles-ci, à leur tour, ont influencé la graphie. Ainsi, les variantes

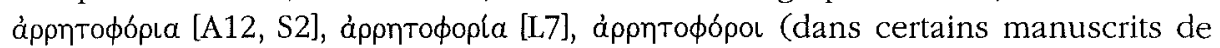
A5), sont apparemment des reconstructions savantes - et tardives - fondées sur

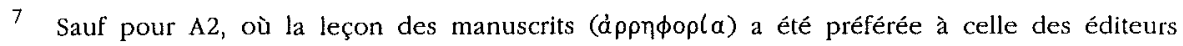

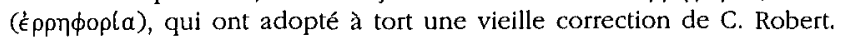

8 Contrairement à ce qu'affirme un peu légèrement BRULÉ, op. cit. (n. 1), p. 79. Cf. L. THREATTE, The grammar of Attic inscriptions, I, Berlin, 1980, p. 127-128.

9 La seule attestation litigieuse est la plus ancienne (A1), où l'augment d'r̉ppnф́b́ovv ne permet pas de trancher entre les deux orthographes possibles. 


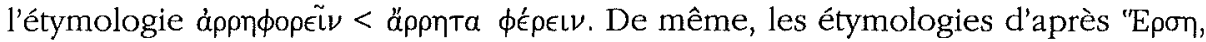
qu'il s'agisse de la fille de Cécrops ou du nom commun désignant la rosée, doivent être responsables des variantes en $\epsilon \rho \sigma \eta$-, ainsi que de l'hésitation des copistes entre l'esprit doux et l'esprit rude sur l' $\epsilon$ - initial. La variante isolée

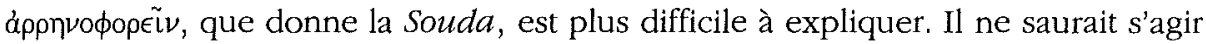
d'un lapsus de copiste, puisqu'elle apparaît à la fois en lemme [L5b], à sa place alphabétique normale ${ }^{10}$, et dans un renvoi [L5e]. Elle pourrait témoigner d'une

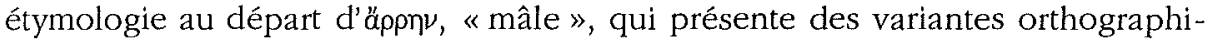

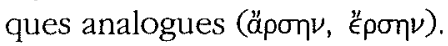

\section{Les données épigraphiques}

Le corpus épigraphique relatif à l'arrhéphorie compte actuellement vingtcinq inscriptions. Vingt d'entre elles sont des dédicaces de portraits d'arrhé-

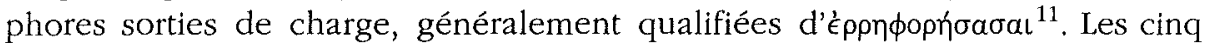
autres sont :

- un décret voté en $137 / 6$ av. J.-C. en l'honneur de Léonidès, qui avait été prêtre d'Asclépios l'année précédente et « avait donné sa fille [-... lors des] Épidauries

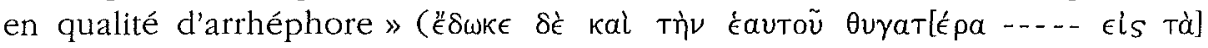

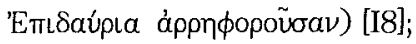

- deux inscriptions, datées du $\mathrm{II}^{\mathrm{e}}$ siècle ap. J.-C., réservant des sièges du théâtre de Dionysos, d'une part, aux deux éponфópol de Chloé Thémis [I22], de l'autre, à celles de l'Ilithye d'Agrai [I23];

- la dédicace, également datée du II ${ }^{\mathrm{e}}$ siècle ap. J.-C., du portrait de son fils par

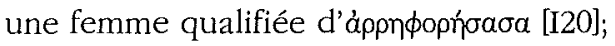

- la dédicace, datée vers 230 ap. J.-C., à Amphiaraos, dans son sanctuaire d'Oropos, de deux statues par les fils d'un ex-archonte éponyme et d'une àppnфópos [I24].

Je n'ai pas retenu :

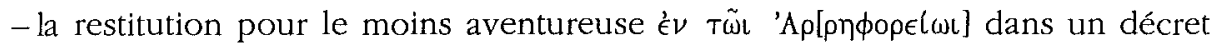
athénien sur les Panathénées, daté vers 335 av. J.-C. ${ }^{12}$;

10 Entre 'A $\rho \eta \dot{\nu \eta}$ et áprí $\nu \omega \rho$, le lexicographe traitant $\rho \rho$ comme s'il s'agissait d'un $\rho$ simple.

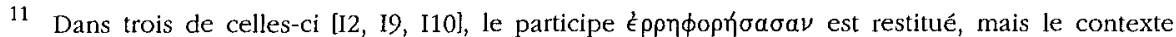
rend la restitution vraisemblable. Dans quatre autres cas [I3, I11, I15, I19], l'initiale n'est pas conservée et une forme en $a$ - serait théoriquement possible, quoique statistiquement peu probable. Au lieu

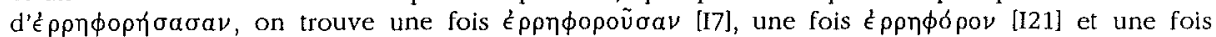

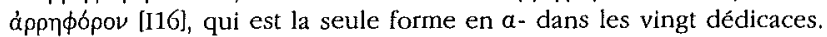

$12 I G, \mathrm{II}^{2}, 334,1.9-10 ;$ Fr. SOKOLOWSKI, Lois sacrées des cités grecques, Paris, 1969, p. 63-66, $\mathrm{n}^{\circ} 33$. La restitution est de S. HUMPHREYS dans The craft of the ancient bistorian. Studies in bonor of C.G.

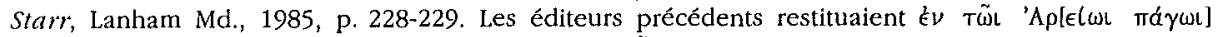

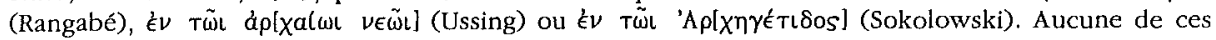
restitutions ne s'impose. 
- une dédicace d'époque antonine copiée à Mytilène par Cyriaque d'Ancône et

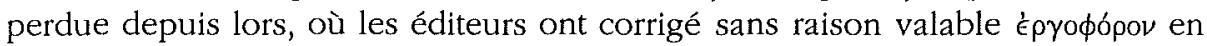

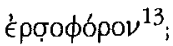

- un décret athénien en l'honneur de Julia Domna, daté de 193-195, dans lequel J.H. Oliver restitue audacieusement $[\theta \dot{u} \epsilon \iota v] \delta \dot{~ k a l ~ t a ̀ s ~[a ̉ \rho \rho \eta \phi o ́ p] o u s ~ T a ̀ s ~}$

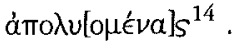

Dix-neuf des vingt bases de portraits d'arrhéphores ont été trouvées sur l'Acropole d'Athènes ou le flanc sud de celle-ci [125]. Les plus anciennes remontent à la seconde moitié du $\mathrm{II}^{\mathrm{e}}$ siècle av. J.-C., les plus récentes au $\mathrm{II}^{\mathrm{e}}$ siècle ap. J.-C. Plusieurs d'entre elles conservent la mention des dédicants. Dans la plupart des cas, il s'agit des parents de l'arrhéphore [I2, I5, 16, I10, I14, I16, I17, I18], auxquels s'associent parfois un oncle [I4] ou des frères [I7, I11, I13, I21]; dans un cas, la mère est l'unique dédicante [I19]; dans un autre, le grand-père se substitue au père, sans doute décédé [I12]. Deux statues sont dédiées par le Conseil et le Peuple [I17, I18]; elles datent l'une et l'autre du i ${ }^{\text {er }}$ siècle ap. J.-C.

Lorsque la dédicace d'un de ces dix-neuf portraits conserve la mention d'une divinité, il s'agit toujours d'Athéna, qualifiée ou non de Polias et éventuellement associée à Pandrose [I6, I11, I14]. On interprète généralement le nom de

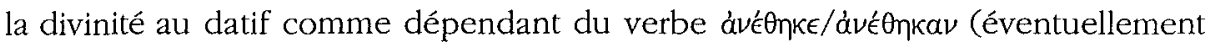
sous-entendu) et désignant la dédicataire. Cette interprétation semble effectivement s'imposer dans les dédicaces antérieures à l'époque impériale romaine. En revanche, dans celle de Tertia [I17], le nom au datif dépend clairement du

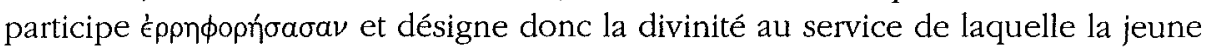
fille a été arrhéphore:

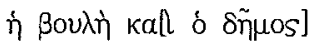

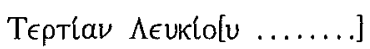

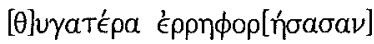

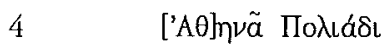

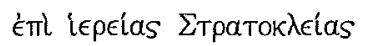

Le Conseil et le Peuple (ont dédié la statue de) Tertia fille de Lucius [-- - ] qui a été arrhéphore pour ( $=$ au service d')Athéna Polias sous la prêtrise de Stratocléia.

Au-dessous, quatre couronnes entourent des inscriptions rappelant que Tertia a été honorée successivement comme arrhéphore, initiée du foyer,

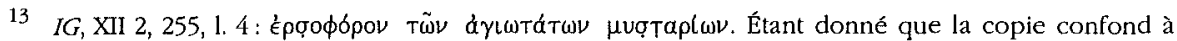
plusieurs reprises $C$ (carré) et $\Gamma$, la correction est paléographiquement possible, mais Époóópos

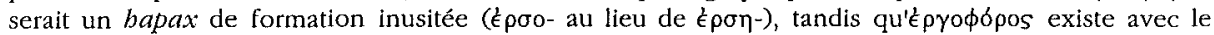
sens d'« abeille ouvrière » (AEliAN., Nat. anim., V, 42) et pourrait avoir désigné métaphoriquement une fonction sacerdotale dans le cadre des mystères des Cabires.

$14 I G, \mathrm{II}^{2}, 1076,1.33-34 ;$ J.H. OLIVER, Atbenian Siudies presented to W.S. Ferguson $=$ HSPb, suppl. 1, Cambridge Mass., 1940 (réimpr. New-York, 1973), p. 521-530. Pour la date, voir A.N. OIKONOMIDES, $B A S P, 21$ (1984), p. 179-180. 
canéphore aux Éleusinies et canéphore aux Épidauries. La dédicace est donc largement postérieure à sa sortie de charge en qualité d'arrhéphore sous la prêtrise de Stratocléia, laquelle est déjà évoquée dans une dédicace datée du $\mathbf{r}^{\mathrm{er}}$ siècle av. J.-C. [I13], alors que celle-ci date du ${ }^{\text {er }}$ siècle ap. J.-C. Il n'empêche que c'est au titre d'ancienne arrhéphore que Tertia se voit élever une statue par la cité. Par la suite, ce titre sera revendiqué par des femmes mariées et mères de

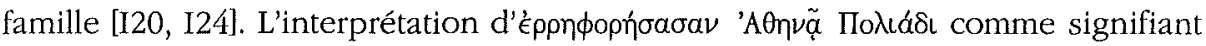
«qui a été arrhéphore au service d'Athéna Polias » est également probable dans l'autre cas de dédicace par le Conseil et le Peuple [I18]. Elle est au moins possible pour I15 et I19.

Dans la dédicace de Tertia, Stratocléia est mentionnée en qualité de prêtresse d'Athéna Polias; on en déduira qu'elle l'était en la même qualité dans celle de Stratonice [I13]. Dans deux ou trois autres cas, nous savons par ailleurs que le nom précédé de la formule émi iepelas est celui d'une prêtresse d'Athéna Polias : cette fonction est attestée pour Chrysis [110] par un décret des Delphiens et la dédicace d'une statue érigée sur l'Acropole ${ }^{15}$ et pour Alexandra [I15] par quatre autres dédicaces ${ }^{16}$; elle le serait également pour Philotéra [I7] si celle-ci est

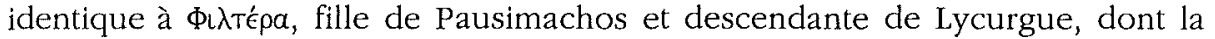
base de la statue par Eucheir et Euboulidès, avec dédicace métrique, a été retrouvée sur l'Acropole et dont la prêtrise est évoquée, en outre, dans la dédicace d'une autre statue érigée à cet endroit ${ }^{17}$. Il est plus que probable qu'il en allait de même des huit autres prêtrises mentionnées $\left[\mathrm{I} 1^{18}, \mathrm{I} 3^{19}, \mathrm{I} 4, \mathrm{I}\right.$, I6, I10, I14]. En outre, les noms de deux arrhéphores, Xénostraté [I7] et Panarista [I11], se retrouvent dans des listes d'ergastines honorées par décret respectivement en 108/7 et en 99/8 av. J.-C. ${ }^{20}$; les ergastines étaient les jeunes filles qui se relayaient pour tisser le voile dont les arrhéphores contribuaient à poser la trame le jour de la fête des Chalkéia. Tous ces éléments, ainsi que la provenance des dix-neuf bases, donnent à penser que les arrhéphores dont elles portaient les statues avaient été au service d'Athéna.

$15 I G, \mathrm{II}^{2}, 1136$ et 3484.

$16 I G, \mathrm{I}^{2}, 3155,4341,4342,4344$.

$17 I G, \mathrm{II}^{2}, 3474$ et 3870. J. KIRCHNER, Propographia Attica, II, Berlin, 1903, $\mathrm{n}^{\circ} 14936$, pensait à Philotéra fille de Nicomachos de Collarges, qui figure parmi les ergastines honorées par un décret voté en $99 / 8\left(I G, I^{2}, 1034 d, 1,11\right)$.

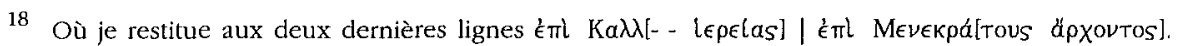

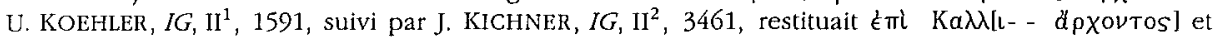
supposait une datation par deux archontes éponymes successifs. Cependant, St. Dow, Hesperia, 2 (1933), p. 437, a montré que Ménécratès fut archonte en 220/19 et Calli[---] en 217/16. Il suggérait

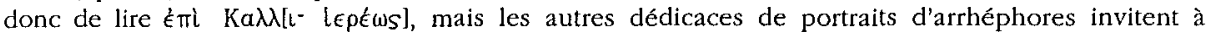
restituer ici la mention d'une prêtrise féminine.

19 Où je suis tenté de restituer aux deux dernières lignes, sur le modèle de $[1$, [éml - - ] $\mu \eta \delta \epsilon$ llas

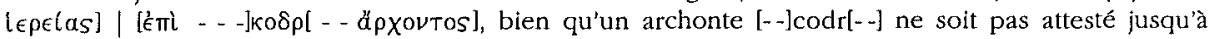
présent au $\mathrm{III}^{\mathrm{e}}$ siècle av. J.-C.

$20 I G, \mathrm{II}^{2}, 1036, \mathrm{l} .37$, et 1034 , d, l. 9. 
Le cas de la dédicace I25 est plus délicat. Pittakis, qui la signale simplement en note à la publication d'une autre inscription, l'a vue il y a un siècle dans une église d'Athènes, où elle n'était certainement pas à son emplacement originel, que nous devons nous résoudre à ignorer. Il la transcrit comme suit:

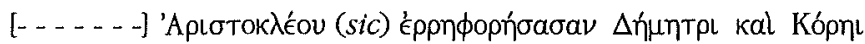

La pierre étant apparemment perdue, l'absence de critères paléographiques empêche de préciser la date de cette dédicace. Toutefois, l'omission du $s$ final

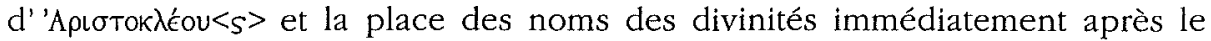

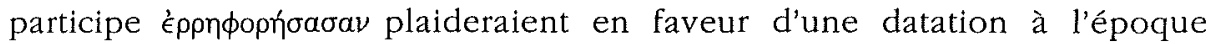
impériale romaine. L'inscription est trop mutilée pour décider si Déméter et Coré sont les dédicataires de la statue d'une arrhéphore d'Athéna Polias ou si l'arrhéphore était à leur service. L'absence d'attestation de portraits d'arrhéphores d'Athéna Polias dédiés à d'autres divinités que cette dernière me ferait pencher en faveur de la seconde hypothèse.

\section{Les données lexicographiques...}

Avatars tardifs de traditions érudites de provenances et de dates diverses, lexiques et scholies se révèlent d'une utilisation particulièrement délicate. Il est d'autant plus regrettable que, depuis les travaux pionniers de R. Reitzenstein ${ }^{21}$, ces sources capitales n'aient pas été suffisamment étudiées pour elles-mêmes et que plusieurs d'entre elles demeurent inédites ou accessibles seulement dans des éditions vieillies. L'analyse que j'en propose doit donc être considérée comme une première esquisse d'une recherche qui reste à approfondir. Pour ce qui concerne l'arrhéphorie, deux groupes principaux de notices lexicales se

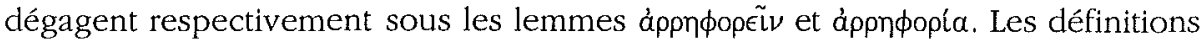
que donnent du premier Harpocration [L1], l'Etymologicum Magnum [L6] ${ }^{22}$, la Souda [L5b] et deux lexiques publiés par Imm. Bekker [L9a, L10] sont d'une telle homogénéité qu'on peut aisément les fusionner en un seul texte que, pour simplifier, j'appellerai La:

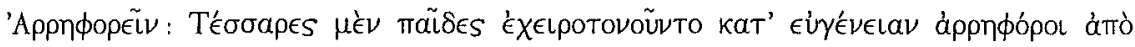

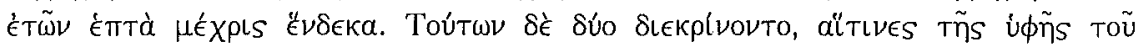

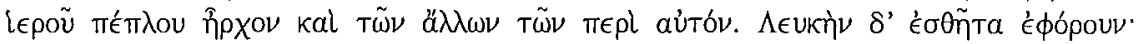

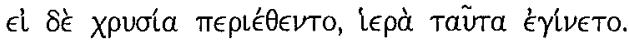

21 Inedita poetarum Graecorum fragmenta, Rostock, 1890/91 et 1891/92; Geschicbte der griecbiscben Etymoligika, Leipzig, 1897 (réimpr. Amsterdam, 1964). Voir, en dernier lieu, la préface de Fr. LASSERRE et N. LIVADARAS éd., Etymologicum Magnum genuinum..., I, Rome, 1976, p. V-XXX.

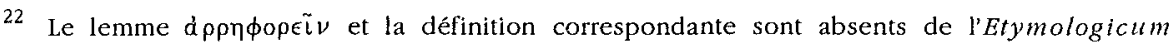
genuinum. 


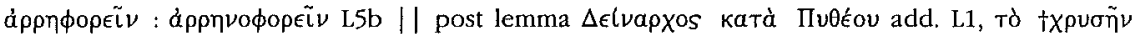

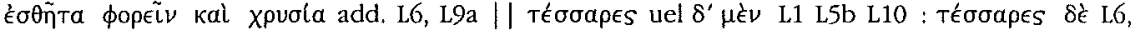

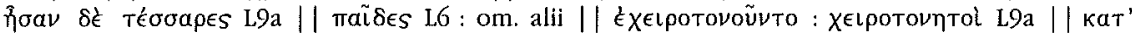

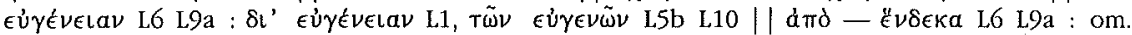

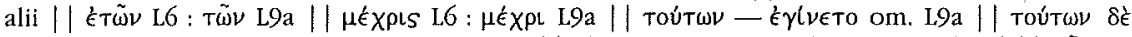

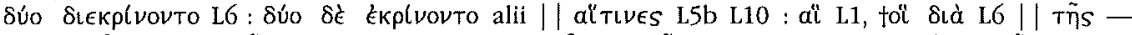

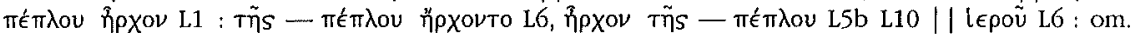

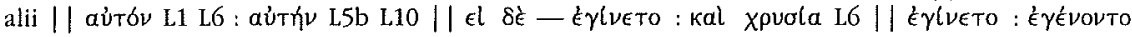
L10.

Être arrbéphore: Quatre fillettes étaient élues d'après leur noblesse arrhéphores de sept jusqu'à onze ans. Deux d'entre elles étaient choisies, lesquelles commençaient le tissage du voile sacré et les autres (rites) qui le ${ }^{23}$ concernent. Elles portaient un vêtement blanc; si elles mettaient des parures d'or, celles-ci devenaient sacrées.

Les notices ayant pour lemme d $\rho \rho \eta \phi o \rho l \alpha$ forment un groupe un peu moins homogène, que j'appellerai $L \beta$. Elles se répartissent en deux branches. $L \beta_{1}$ est attestée par l'Etymologicum genuinum [L4a], la Magna grammatica [L7] et le pseudo-Hérodien [L8]:

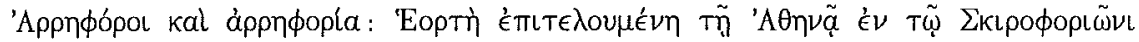

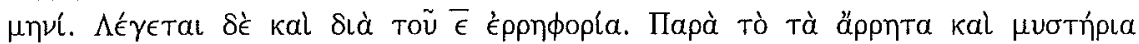

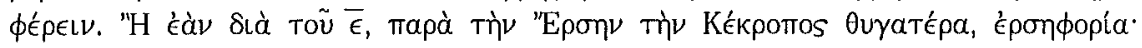

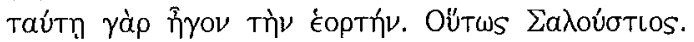

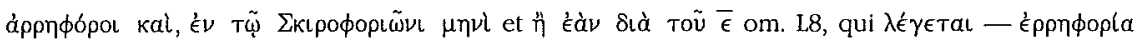

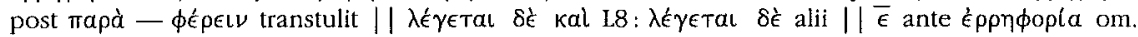

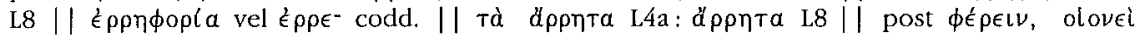

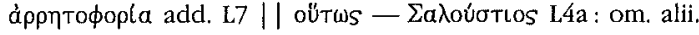

$\mathrm{L} \beta_{2}$ nous est transmise en des termes quasi identiques par la Souda [L5c] et la scholie au vers 642 de la Lysistrata d'Aristophane [S1]:

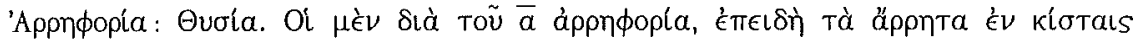

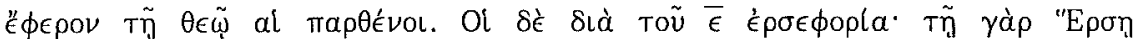

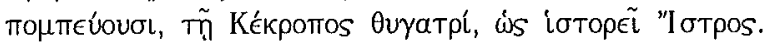

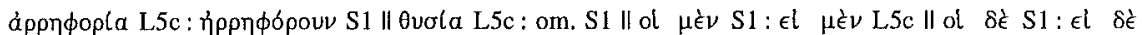

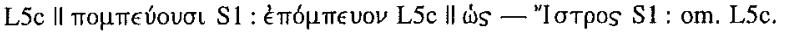

Hésychius [L3a] en donne un résumé :

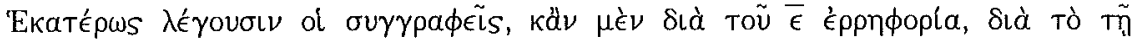

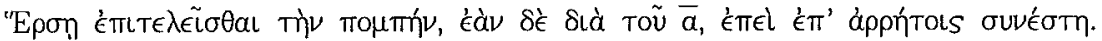

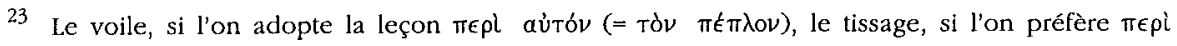

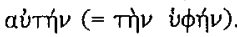


La confrontation des deux versions, $L \beta_{1}$ et $\mathrm{L} \beta_{2}$, ainsi reconstituées montre sans équivoque qu'elles dérivent d'une source commune.

ц 1

Arrbéphores et arrbéphorie:

(1) Fête célébrée en l'honneur d'Athéna au mois de scirophorion.

(2) On dit aussi errbéphorie avec $e$. (Ainsi appelée) d'après le fait de porter les objets secrets (arrbèta) et mystérieux. Ou, si c'est avec $e$, d'après Hersé, la fille de Cécrops, (c'est-à-dire) (b)erséphorie, car c'est en l'honneur de celle-ci qu'on organisait la fête. C'est ce que dit Saloustios.
$\mathbf{L} \mathbf{3}_{2}$

Arrbéphorie:

(1) Sacrifice.

(2) Les uns (écrivent) arrbéphorie avec a parce que les jeunes filles portaient les objets secrets (arrbèta) dans des cistes en l'honneur de la déesse. Les autres (écrivent) erséphorie avec $e$, car elles font la procession en l'honneur d'Hersé, la fille de Cécrops, comme le rapporte Istros.

En effet, les deux versions se complètent et s'expliquent l'une l'autre. Chacune d'elle s'articule en deux parties: (1) définition du mot áppnфopía; (2) justification étymologique des deux orthographes du mot. $L \beta_{2}$ réduit la première à un laconique $\theta v \sigma i a(=\dot{\epsilon}$ optŕ), éludant le nom de la déesse $(\tau \tilde{n} \theta \in \tilde{\omega})$ en l'honneur de laquelle les jeunes filles portent les arrbèta et que nous connaissons grâce à $\mathrm{L} \beta 1$. À l'inverse, dans la seconde, $\mathrm{L} \beta 1$ omet les jeunes filles, les cistes, la déesse elle-même et remplace la mention de la procession (

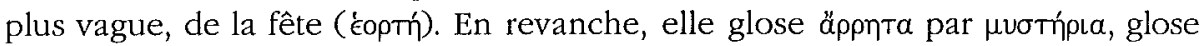

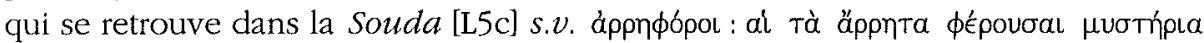
et, sous une forme légèrement différente, dans un autre lexique [L11] s.v.

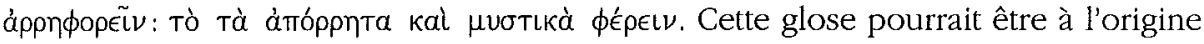

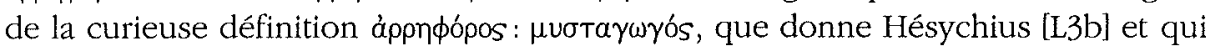

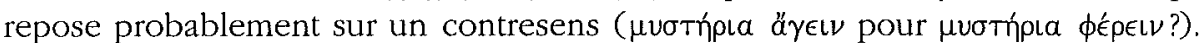

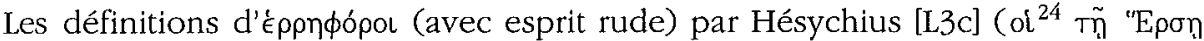

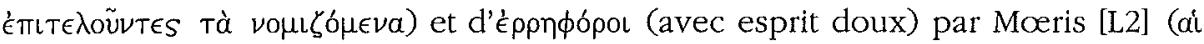

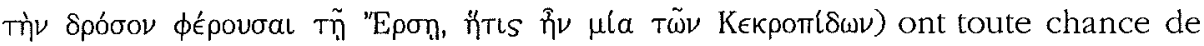
puiser à la même source: la première se fonde sur l'étymologie par Hersé; la

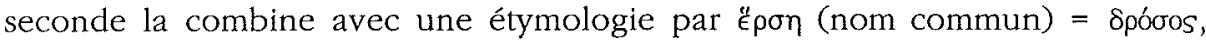
« rosée », et rappelle la filiation d'Hersé.

D'autre part, on peut rapprocher de La les notices identiques de l'Etymologicum genuinum [L4b] et de la Souda [L5f] sur la fête des Chalkéia, où la participation des arrhéphores au tissage du voile sacré est évoquée de façon plus précise :

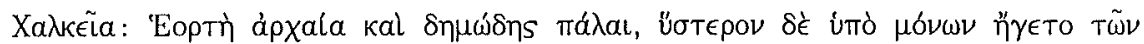

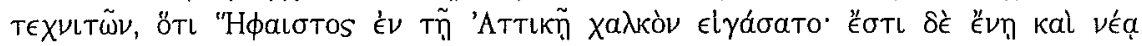

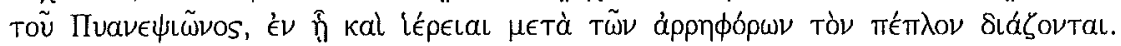

24 L'emploi de ol comme article défini féminin pluriel, qui est normal en grec moderne, pourrait remonter au Moyen âge et avoir influencé la graphie des copistes. 
Chalkéia: Fête ancienne et autrefois publique, elle fut célébrée ensuite par les seuls artisans, parce qu'Héphaistos a travaillé le bronze en Attique. Elle a lieu le dernier jour de pyanepsion, qui est aussi le jour où les prêtresses posent la trame du voile avec les arrhéphores.

\section{... et leurs sources}

L'ensemble de la tradition lexicographique relative à l'arrhéphorie offre une cohérence remarquable, qui laisse supposer qu'elle dérive d'une source unique. Pour La, Harpocration [L1] cite Dinarque, orateur athénien du tournant des $\mathrm{IV}^{\mathrm{e}}$ et III $^{\mathrm{e}}$ s. av. J.-C., « dans son discours Contre Pytbéas », mais cette référence ne doit pas faire illusion: elle signifie seulement que l'orateur avait utilisé le mot

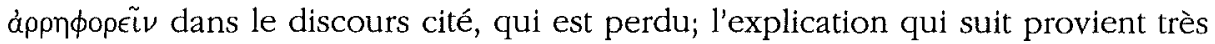
certainement d'une autre source. $\mathrm{L} \beta 1$ renvoie explicitement à Saloustios, un grammairien qui vécut à une date indéterminée entre le II $^{\mathrm{e}}$ et le $\mathrm{V}^{\mathrm{e}}$ siècle ap. J.-C. ${ }^{25}$, tandis que $\mathrm{L} \beta_{2}$ invoque l'autorité d'Istros - sans préciser, il est vrai, si c'est pour la totalité de la notice, la seule étymologie d'éppnфopía ${ }^{26}$ ou unique ment la filiation d'Hersé.

Sans doute originaire du nord de la Grèce, Istros fut « esclave et familier » du fameux érudit alexandrin Callimaque ( III $^{\mathrm{e}} \mathrm{s}$, av, J.-C.) ${ }^{27}$. Il pourrait avoir séjourné auparavant à Athènes, dont il connaît, en tout cas, la topographie et au passé de laquelle il porte un intérêt évident. Parmi ses nombreuses compilations,

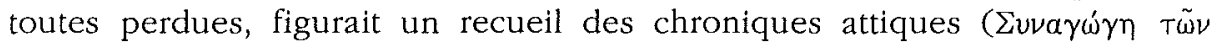
'Aт $\theta(\delta \omega \nu)$ en au moins quatorze livres, dont quelques fragments sont conservés. On y trouve plusieurs allusions à des faits religieux athéniens - entre autres, la procession aux flambeaux lors des Apatouries, l'appellation primitive des Panathénées, l'institution des oschophores («porteurs de sarments de vigne ») par Thésée «à raison de deux parmi (les Athéniens) qui l'emportaient par la

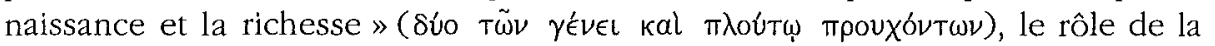
trapézophore, sorte de coadjutrice de la prêtresse d'Athéna ${ }^{28}$. Ailleurs, il évoquait l'origine du rite du pharmakos ou celle de l'épithète Tritogénie appliquée à Athéna ${ }^{29}$. Dans un tel contexte, des considérations sur l'arrhéphorie n'auraient rien d'inattendu.

Les compilations d'Istros offraient une mine de renseignements aux commentateurs soucieux d'éclairer les allusions des orăteurs attiques « classiques » aux institutions religieuses de leur cité. Harpocration s'y réfère une bonne dizaine de fois et, même si la notice $\mathrm{LI}$ ne l'invoque pas explicitement, il est très

25 Sur Saloustios, voir R. PFEIfFer éd., Callimachos, II, Oxford, 1953, p. XXVIII-XXX.

26 Comme le pense PFEIFFER, op. cit. (n. 25), I, 1949, p. 470 (ad fr. 741).

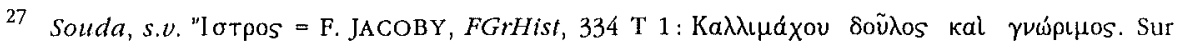
Istros, v. ibid., IIIb, Suppl., Leyde, 1954, p. 618-627.

28 Apatouries : F 2; Panathénées: F4; oschophores : F 8; trapézophore: F 9 ( $c f$. ci-desssous n. 36).

29) Tritogénie: F 24; pharmakos : F 50. 
probable qu'elle puise, elle aussi, à cette source, qui serait donc celle de L $\alpha$. D'autre part, il serait étonnant qu'Istros, source au moins partielle de L $\beta 2$, n'ait pas inspiré, directement ou indirectement, la source de $L \beta 1$. De fait, Saloustios était notamment l'auteur d'un commentaire d'Hécalé, célèbre poème du maître d'Istros, Callimaque, dont il subsiste un certain nombre de fragments. Leur éditeur, R. Pfeiffer, considère que L4a, c'est-à-dire L $\beta_{1}$, est emprunté à ce commentaire, auquel l'Etymologicum genuinum et la Souda se réfèrent volontiers ${ }^{30}$. Or, où les exégètes d'Hécalé avaient-ils le plus de chances de trouver les clés d'une ouvre ayant pour cadre l'Attique des temps légendaires et truffée d'allusions à des mythes peu connus et à des rites insolites sinon chez Istros? D'autant qu'on peut supposer que ce dernier avait aidé son maître à en rassembler les matériaux.

Pastiche savant de l'épopée traditionnelle, dans laquelle les mythes étiologiques jouent un rôle primordial, Hécalé relatait la fondation par Thésée du sanctuaire de Zeus Hécaléios à la mémoire d'une charmante vieille dame du nom d'Hécalé, chez qui le héros avait reçu l'hospitalité à la veille d'affronter le taureau de Marathon et qu'il avait trouvée morte à son retour. Divers autres mythes émaillaient le récit. Ainsi, au cours de la nuit qui suivit la victoire sur le taureau, deux corneilles évoquaient les punitions infligées à leurs ancêtres pour avoir été messagères de mauvaises nouvelles: Athéna, furieuse d'apprendre que les filles de Cécrops lui avaient désobéi en ouvrant la ciste où le petit Érichthonios était caché, les avait exclues de l'Acropole, tandis qu'Apollon, à l'annonce de l'infidélité de Coronis, avait teint leur beau plumage blanc d'origine en noir. À cette occasion, Callimaque donnait sa version de la conception et de la naissance du « roi serpent » et proposait une étymologie de son nom ámò tõ̃ éplov

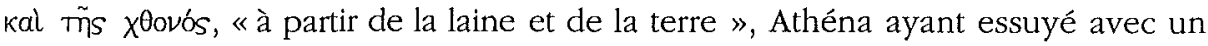
morceau de laine le sperme éjaculé sur sa cuisse par Héphaistos, qui tentait de la violer, et l'ayant jeté à terre ${ }^{31}$.

Selon toute vraisemblance, le poète avait emprunté l'histoire d'Hécalé à l'atthidographe Philochore (fin $\mathrm{IV}^{\mathrm{e}} /$ début $\mathrm{III}^{\mathrm{e}}$ siècle av. J.-C.) et celle des corneilles exclues de l'Acropole à un autre atthidographe, Amélésagoras ${ }^{32}$. Il serait étonnant qu'il n'ait pas utilisé à cette occasion le recueil des chroniques attiques compilé par son collaborateur Istros. La même remarque vaut pour le poème qu'il avait consacré au rite du pharmakos, rite au sujet duquel Istros rapportait un mythe étiologique dans son traité sur les Apparitions d'Apollon ${ }^{33}$. Pour en revenir à l'arrhéphorie, quelques fragments conservés d'Hécalé offrent des parallèles troublants avec la tradition lexicographique qui la concerne. Ainsi les vers 18-21 du fragment $260 \mathrm{Pf}$, extrait du récit des corneilles:

30 PFEIFFER, op. cit. (n. 25), II, p. XXIX.

31 Schol. AD in Iliad., II, 547. Cf. PFEIFFER, op. cit. (n. 25), 1, p. 248 (ad fr. 260, 19).

32 PHILOCHOR., FGrHist, $328 \mathrm{~F}$ 109; AMELESAGORAS, FGrHist, $330 \mathrm{~F}$ 1. PFEIFFER, op. cit. (n. 25), I, p. 227 ( $\mathrm{ad} \mathrm{fr}, 230$, c) et 248-249 ( $\mathrm{ad}$ fr. $260,27 \mathrm{sq}$ ).

33 CALLIM., fr. 90 Pf; ISTROS, FGrHist, 334 F 50. 


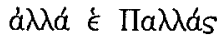

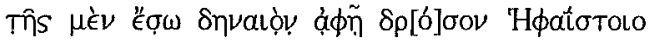

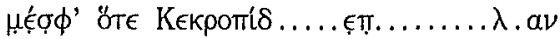

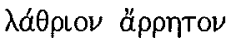

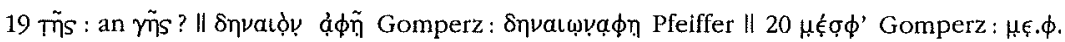

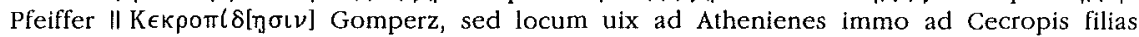
pertinere recte monet Pfeiffer.

... mais Pallas l'a laissé (= Érichthonios) longtemps à l'intérieur (de la ciste?), rosée (= rejeton ${ }^{34}$ ?) d'Héphaistos, jusqu'à ce que les filles de Cécrops ... secret clandestin...

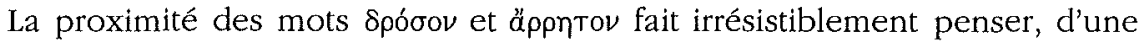

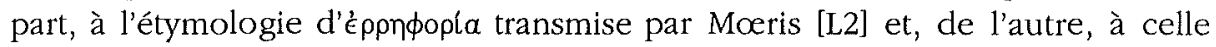
d'áppnфopla selon $\mathrm{L} \beta$ et les notices apparentées.

Un autre vers de Callimaque [fr. $520 \mathrm{Pf}$ évoque la pose de la trame du voile sacré d'Athéna en des termes qui rappellent à la fois L4b/L5f (Tòv Té $\pi \lambda o v$

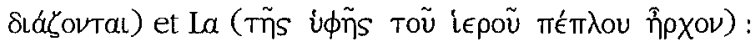

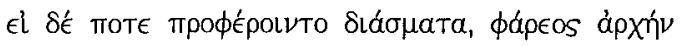

si jamais elles posaient la trame, commencement du voile $35 \ldots$

Pfeiffer penche pour l'attribution de ce vers à Hécalé et y verrait volontiers une allusion à la fête des Chalkéia. À cette occasion, on l'a vu, les artisans honorent en ville leur patron Héphaistos, tandis que, sur l'Acropole, «les prêtresses posent la trame du voile en compagnie des arrhéphores ». Le pluriel lépetar a intrigué les commentateurs modernes pour qui le tissage du voile serait de la compétence de la seule prêtresse d'Athéna. La clé ne se trouverait-elle pas, une fois de plus, chez Istros qui affirmait, après l'orateur Lycurgue, que deux autres

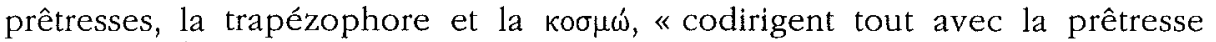
d'Athéna ${ }^{36}$ ?

Ainsi, l'étude des sources des notices lexicographiques relatives à l'arrhéphorie nous ramène chaque fois à Istros. Celui-ci était, semble-t-il, bien au fait des réalités athéniennes de son temps et avait accès aux chroniques originales des atthidographes des $\mathrm{IV}^{\mathrm{e}}$ et $\mathrm{III}^{\mathrm{e}}$ siècle av. J.-C. Dans la mesure où il est à l'origine des données relatives à l'arréphorie, celles-ci sont donc dignes de confiance. Il en va de même du témoignage de Tryphon [A6], relayé par la Souda [L5a], selon

Cf. AESCHYL., Agam., 141.

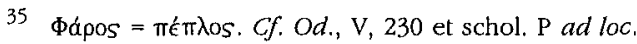

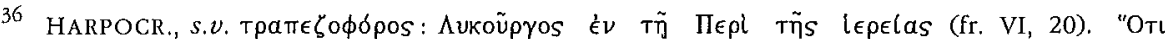

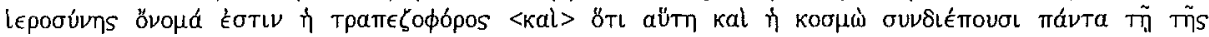

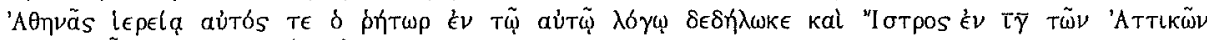

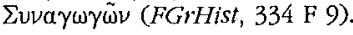


lequel des gâteaux appelés anastatoi étaient faits pour les arrhéphores. Bien qu'il ait vécu deux siècles après Istros, ce grammairien encyclopédique appartenait comme lui au milieu des érudits qui gravitaient autour de la bibliothèque d'Alexandrie et avait accès à des informations de première main sur les réalités cultuelles - et gastronomiques - d'Athènes.

Les étymologies elles-mêmes, pour fantaisistes qu'elles nous paraissent, méritent considération. Des étymologies de ce type se rencontrent dès l'aube de la littérature grecque et il serait fallacieux de les juger selon les critères de la linguistique moderne. Il s'agit plutôt de procédés exégétiques, qui nous renseignent sur la signification et les connotations de tel ou tel mot dans l'esprit de leurs auteurs. Subjectives, elles se fondent néanmoins sur des faits objectifs sous

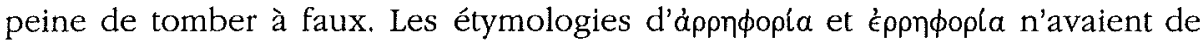
sens pour les contemporains d'Istros et consorts que par rapport à des réalités cultuelles connues de tous. La coexistence de deux orthographes, dont elles prétendaient rendre compte, est d'ailleurs, elle aussi, une réalité, comme l'attestent les inscriptions.

Il ne faut cependant pas perdre de vue que les lexicographes ont puisé l'essentiel de leurs informations dans des commentaires ou dans des lexiques antérieurs, dont les versions successives apparaissent de moins en moins complètes et précises au fur et à mesure qu'elles s'éloignent de la source originale. Il

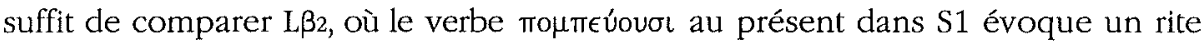
encore en usage, à $\mathrm{L} \beta 1$, qui est entièrement rédigé au passé, ou encore $\mathrm{L} 4 \mathrm{~b} / \mathrm{L} 5 \mathrm{f}$,

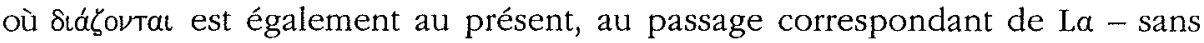
parler de l'arrhéphore-mystagogue d'Hésychius [L3a]. D'autre part, il va de soi que les commentateurs n'ont retenu que les éléments dont ils avaient besoin pour expliquer les obscurités des œuvres qu'ils commentaient. Dans ces conditions, il est possible que les arrhéphores dont ils parlent n'étaient pas les seules à porter ce titre et que les fonctions qu'ils leur prêtent n'étaient pas les seules dont elles fussent investies, ni peut-être même les principales.

\section{Le scholiaste de Lucien}

Avec le scholiaste de Lucien [S2], nous effectuons un saut dans le temps sans quitter pour autant le milieu érudit. La parenté de son témoignage avec celui de Clément d'Alexandrie [A12] le situe, en effet, sans ambiguïté dans la mouvance des intellectuels chrétiens des premiers siècles de notre ère, qui considèrent les rites païens de l'extérieur, sans complaisance, et ne s'estiment plus liés par l'interdiction de divulguer certains de leurs aspects. L'un et l'autre

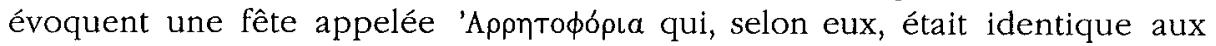
Thesmophories et aux Scirophories et commémorait l'enlèvement de Perséphone par Hadès. Pour Clément, " les femmes célèbrent cette histoire fabuleuse par des fêtes différentes selon les cités, Thesmophories, Scirophories, Arrhéto-

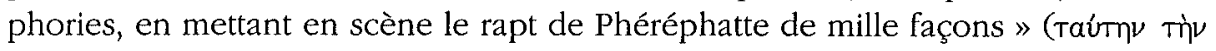

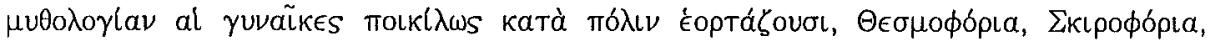




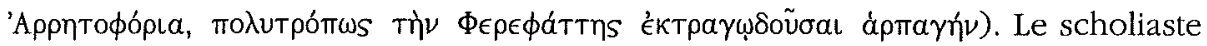
est plus disert. Après avoir affirmé que les Scirophories sont un autre nom des Thesmophories et avoir décrit un des rites de celles-ci ${ }^{37}$, il ajoute: « La même fête est aussi appelée Arrbétophories [...]. À cette occasion aussi, on porte des objets sacrés secrets, façonnés en pâte à pain et représentant des serpents et des organes masculins. On reçoit, en outre, des rameaux de pin à cause de la grande

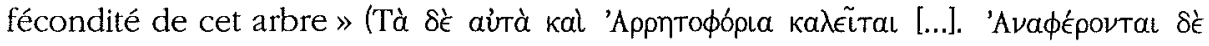

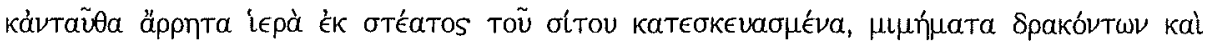

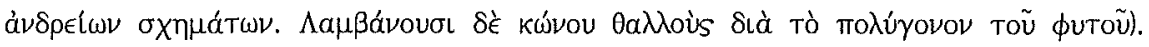

À l'instar de Clément dévoilant le contenu des cistes utilisées lors les mystères ${ }^{38}$, le scholiaste entend révéler la nature des objets secrets portés lors des Arrhétophories. Une fête de ce nom n'est pas attestée en dehors des deux témoignages précités, sauf si on l'identifie à celle que les lexicographes appellent

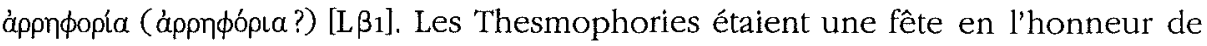
Déméter Thesmophoros, dont la célébration est attestée dans plusieurs cités grecques; à Athènes, elle avait lieu du 11 au 13 pyanepsion (octobre-novembre), avant les semailles. Les Scirophories, qui ne sont pas connues en dehors d'Athènes, y étaient célébrées en l'honneur de Déméter et Coré le 12 scirophorion (juin-juillet), mois auquel elles donnaient leur nom. Ces deux fêtes ont en commun d'être consacrées à Déméter. En revanche, celle que les lexicographes évoquent s.v. ảppnфopla l'était à Athéna [L $\beta_{1}$ ]. Le rapprochement avec les Thesmophories pourrait néanmoins avoir été suggéré par l'usage, dans les deux cas, de figurines façonnées ék oт́́atos toũ oitov, littéralement « en pâte de blé »-ce blé dont Déméter a enseigné la culture aux hommes -, ainsi que de symboles de fécondité ${ }^{39}$.

On a dénoncé le syncrétisme abusif qui inspire de tels rapprochements. Il est cependant conforme aux tendances exégétiques qui se font jour dans le paganisme finissant. Pas plus que les étymologies fantaisistes qui s'y mêlent ne doivent occulter la fiabilité générale des informations que les lexicographes ont empruntées à l'érudition hellénistique au sujet de l'arrhéphorie, le syncrétisme dont le scholiaste de Lucien fait preuve n'autorise à suspecter son témoignage. La concordance de ce dernier avec celui de Clément d'Alexandrie, que l'on tient d'habitude pour une source particulièrement bien documentée, nous incite, au contraire, à l'accepter comme véridique - en tenant compte qu'il émane d'un auteur chrétien de la fin de l'époque impériale.

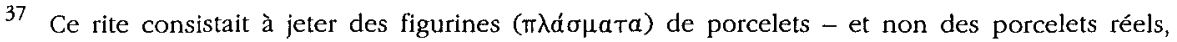

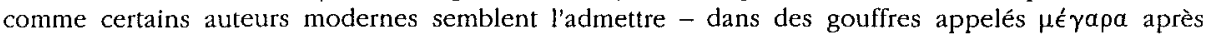
avoir récupéré les restes de celles qu'on y avait jetées l'année précédente, lesquels mélangés à la semence passaient pour garantir une bonne récolte.

CLEM. ALEX., Protr., II, 22, 4-5.

Outre l'existence possible d'arrhéphores de Déméter et Coré [I25]. 


\section{Les sources littéraires}

A la différence des lexiques, les sources littéraires nous apportent des informations essentiellement ponctuelles et, le plus souvent, allusives. La plus ancienne est Aristophane [A1] qui, dans Lysistrata, représentée en 411 av. J.-C., met en scène un chœur de vieilles Athéniennes. Parodiant les discours des orateurs masculins à l'Assemblée, chaque choreute s'adresse au public à la première personne du singulier et proclame son intérêt pour la cité, qui l'a

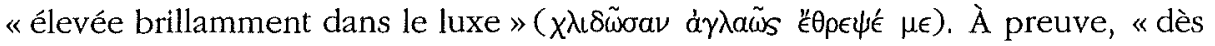

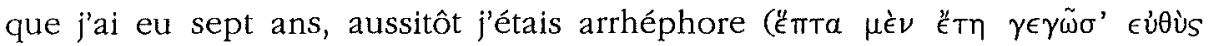

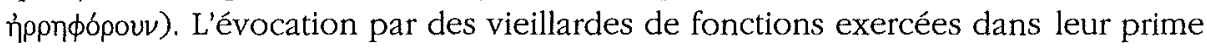
enfance visait, sans aucun doute, à l'effet comique.

Quelques années plus tard, dans un discours écrit par Lysias pour un Athénien accusé de corruption [A2], celui-ci évoque, parmi les liturgies qu'il a assumées, "des archithéories ${ }^{40}$, des arrhéphories et d'autres choses du même genre, pour lesquelles (il a) dépensé plus de trente mines » (àpx $1 \theta \epsilon \omega \rho$ ías kal

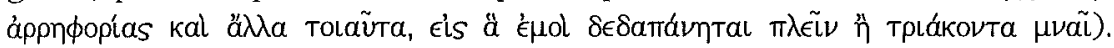

Selon la Vie d'Isocrate attribuée à Plutarque [A9], d'aucuns prétendaient qu'une statue de bronze érigée dans le jeu de balle ( $\sigma \phi \alpha i p l \sigma \tau \rho \alpha)$ des arrhéphores représentait l'orateur «à cheval quand il était encore enfant » ( $\kappa \in \lambda \eta T i \zeta \omega \nu$ Ë TL maĩs $\ddot{\omega} v)$. Le jeu de balle se trouvait sans doute sur l'Acropole, où résidaient deux arrhéphores attachées au service d'Athéna Polias, comme on verra. La statue devait être un ex-voto d'un genre assez courant: Pline l'Ancien mentionne des celtizontes pueri en bronze parmi les œuvres des sculpteurs Canachos de Sicyone et Hégias d'Athènes, l'un et l'autre actifs au tournant des $\mathrm{vl}^{\mathrm{e}}$ et $\mathrm{v}^{\mathrm{e}}$ siècle av. J.-C. ${ }^{41}$

Le fait qu'une comédie de Ménandre [A4] est intitulée tantôt 'Appnфópos ou

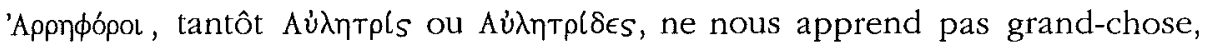
d'autant qu'il n'est question nulle part d'arrhéphores dans les quelques fragments conservés, ni d'ailleurs de joueuses d'aulos. Tout au plus peut-on supposer que l'auteur tirait parti du contraste entre la vie dissolue des secondes et la pureté exigée des premières, comme l'attestent d'autres sources.

Un dialogue anonyme, partiellement conservé sur papyrus [A3], retiendra davantage notre attention. On en a cherché l'auteur dans l'entourage d'Aristote; il pourrait s'agir d'Héraclide du Pont. Il évoque, entre autres, l'idylle du jeune Thrasybule, fils de Philomèle, encore adolescent ( $\mu \epsilon \iota p a ́ k ı \nu)$, avec la plus jeune fille de Pisistrate, dont il est tombé amoureux en la voyant àppnфopoũ $\sigma \alpha \nu$. Une lacune nous prive de la suite, que des sources plus tardives nous font con-

40 L'archithéorie consistait à diriger une délégation officielle à des jeux ou à d'autres cérémonies religieuses panhelléniques et à en supporter les frais.

41 Nat. bist., XXXIV, 75 et 78. G. P. OrKONOMOS, $A E, 6$ (1920-1921), p. 59, a proposé de corriger dans ces deux passages celetizontes en ceretizontes ( jouant à la balle »), sans raison valable. 
naître ${ }^{42}$. Ayant embrassé la fillette en public, le jeune homme est amené devant le tyran qui, au lieu de le mettre à mort pour ce crime comme le réclame son entourage, lui pardonne en disant: "Si nous haïssons ceux qui nous aiment ${ }^{43}$, que ferons-nous à ceux qui nous haïssent » et lui donne sa fille en mariage. Ces sources semblent néanmoins télescoper la rencontre qui provoqua le coup de foudre et la scène du baiser: Diodore raconte qu'un jeune homme, qu'il ne nomme point, embrassa la fille de Pisistrate alors qu'elle était canéphore (кavmpopoírns); Polyen, qui appelle le jeune homme Thrasymède, la lui fait embrasser

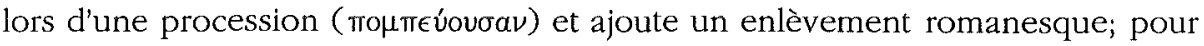
Valère Maxime et Plutarque, le nom du protagoniste est bien Thrasybule, mais ils ne précisent pas les circonstances du baiser. Il est clair que la préférence du philologue doit aller à la version du papyrus, qui est la plus ancienne et semble avoir été aussi la plus détaillée ${ }^{44}$.

Diodore confond ici arrhéphore et canéphore. De même, Denys d'Halicarnasse [A5] compare la fonction des tutulatae romaines - sur laquelle nous ne sommes malheureusement guère renseignés - à la fois à celle des canéphores et à celle des arrhéphores grecques. Il faut croire que les deux fonctions avaient quelque chose en commun, à savoir participer à des processions.

Selon le grammairien Tryphon [A6], dont il a été question plus haut, et la Souda [L5a], on faisait pour les arrhéphores un gâteau appelé anastatos. Le nom, interprété un peu vite comme «le dressé », "l'érigé », a fait rêver certains exégètes modernes de phallus en érection ${ }^{45}$. Certes, le scholiaste de Lucien [S2] prétend que des objets secrets en forme d'organes masculins étaient portés lors des Arrhétophories. Mais, même si celles-ci étaient bien la fête athénienne mentionnée par les lexiques s.v. áppnфopla, le secret était toujours de mise au temps de Tryphon. Dès lors, les anastatoi - quel que soit le sens qu'il faille donner à leur nom - ne sauraient être des figurines que les arrhéphores auraient portées à leur insu, mais tout simplement des gâteaux qu'elles pouvaient

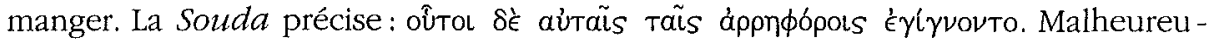
sement, le sens de aủtaĩs n'est pas clai $\mathbf{r}$ : «même pour les arrhéphores », c'est-à dire que les arrhéphores pouvaient en manger aussi? « en propre pour les arrhéphores ", c'est-à-dire que ce type de gâteau leur était réservé? Dans les deux cas, l'allusion à un régime alimentaire particulier est l'hypothèse la plus plausible. Ce n'est peut-être pas un hasard si, dans la Souda, la définition des anastatoi est suivie immédiatement de l'évocation d'une autre sorte de gâteaux, les cbarisioi,

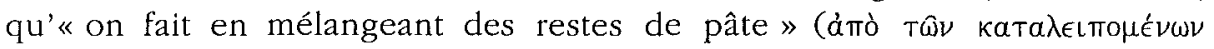

42 DIOD., IX, 37, 1; VAL. MAX., V, 1, 2; PLUT., Mor., 189c et 457f; POLYAEN., Stratag., 5, 14.

43 Jeu de mots sur les deux sens de $\phi(\lambda \in \tilde{\imath} \nu$, « embrasser » et «aimer ».

44 BRULE, op. cit. (n. 1), p. 288, qui préfère la version de Diodore parce qu'elle s'accorde mieux

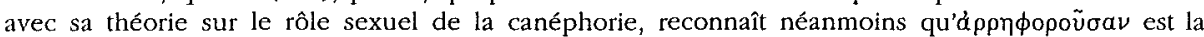
lectio difficilior.

45 Notamment BRULÉ, op. cit. (n. 1), p. 92. 


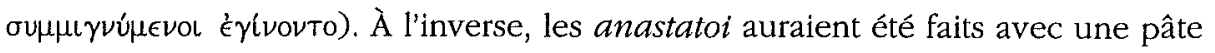
« dépourvue » (autre sens d'ádúatatos) de toute impureté.

D'autres sources semblent d'ailleurs indiquer que les arrhéphores étaient soumises à des règles de pureté rituelle particulièrement strictes. Ainsi, Philon

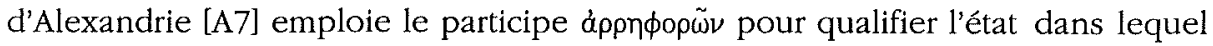
le grand-prêtre d'Israël doit se trouver pour pénétrer dans la Tente du Rendezvous ou s'approcher de l'autel des sacrifices. D'après le contexte, il s'agit d'un état de pureté religieuse parfaite. La pureté des arrhéphores était donc un lieu commun, ce que confirme un fragment d'Élien [A10], où des arrhéphores sont associées à des «femmes tout à fait pures »:

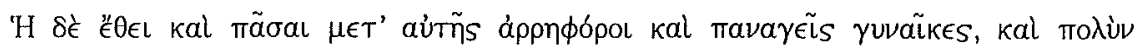

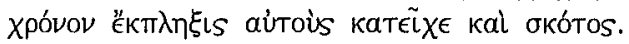

Elle courait et, avec elle, toutes les arrhéphores et les femmes tout à fait pures, et pendant un long moment l'épouvante et l'ombre les (= les spectateurs) retenaient.

On ignore de quelle ouvre est extraite cette citation, dont le contexte est obscur. Il s'agit apparemment d'une scène mythique au cours de laquelle des personnages masculins sont saisis d'effroi à la vue d'un personnage féminin (héroine ou déesse?) en train de courir (de s'enfuir?) en compagnie d'arrhéphores et de femmes tout à fait pures.

J'attribuerais volontiers au même auteur une citation également énigmatique [A11], que transmettent la Souda et l'Etymologicum Magnum ${ }^{46}$ :

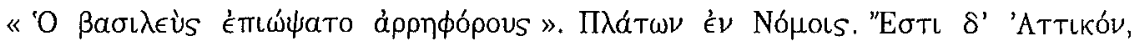

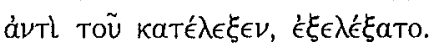

«Le roi a porté le regard sur des arrhéphores ». Platon dans les Lois. C'est une forme attique, au lieu de « il a sélectionné, il a choisi ».

Comme la citation ne se trouve pas dans l'œuvre du philosophe, on en a tiré argument pour l'attribuer à l'auteur comique homonyme. Mais le verbe émเółoua est bel et bien utilisé dans les Lois $(12,947 \mathrm{c})$ avec le sens en question. La citation doit donc provenir d'une autre source, non précisée. Or, la Souda cite souvent Élien sans le nommer et le recours à des formes ou des sens rares est fréquent chez cet atticiste impénitent.

\section{Le témoignage de Pausanias}

J'ai gardé pour la fin le témoignage de Pausanias [A8], non seulement parce qu'il a particulièrement retenu l'attention des exégètes, mais aussi parce que,

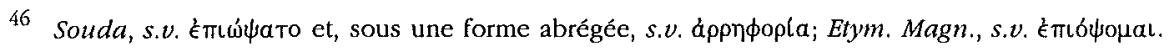


malgré cela, il continue de poser des problèmes de lecture, de traduction et d'interprétation.

Décrivant l'Acropole d'Athènes, le périégète mentionne le Pandroséion, puis, enchaîne sans transition:

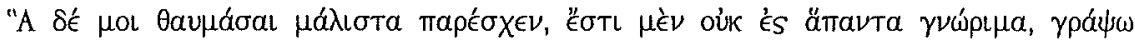

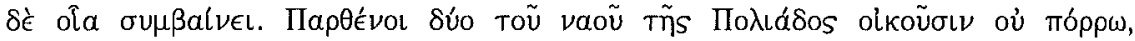

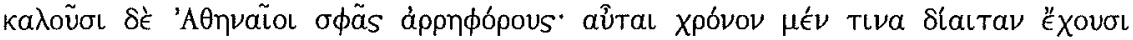

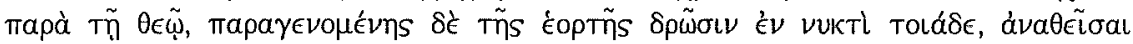

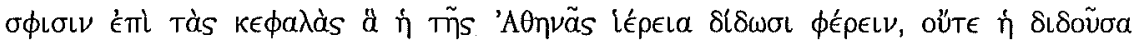

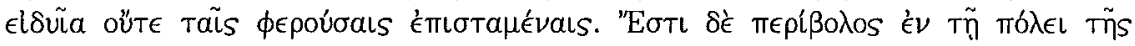

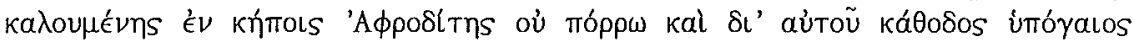

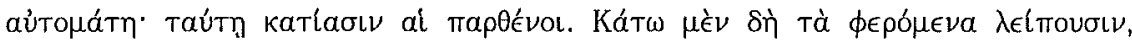

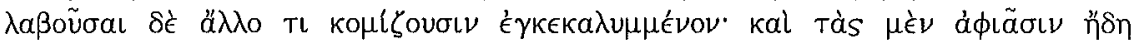

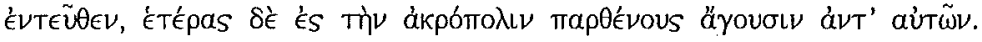

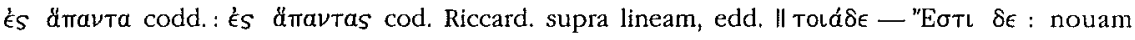
punctuationem proposui.

Ce qui a été pour moi le plus grand sujet d'étonnement ne peut être divulgué entièrement, mais je décrirai néanmoins à peu près comment cela se passe. Deux jeunes filles habitent non loin du temple d'(Athéna) Polias; les Athéniens les appellent arrbépbores. Ces jeunes filles séjournent un certain temps auprès de la déesse et, au moment de la fête, elle accomplissent de nuit le rite suivant, après avoir posé sur leur tête les objets que la prêtresse d'Athéna leur donne à porter, sans que celle qui les donne sache (ce qu'elle donne) ni que celles qui les portent en aient connaissance. Il y a en ville, non loin (du sanctuaire) de l'Aphrodite appelée "dans les jardins", un enclos sacré dans lequel pénètre une descente souterraine naturelle; c'est par là que les jeunes filles descendent. Une fois en bas, elles abandonnent ce qu'elles portaient et reçoivent un autre objet qu'elles emportent enveloppé. Dès cet instant, on les libère et on conduit d'autres jeunes filles sur l'Acropole à leur place.

En ce qui concerne le texte, je rétablis, avec W. Burkert ${ }^{47}$, la leçon És

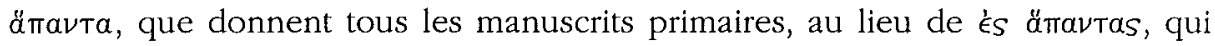
n'est qu'une correction supralinéaire d'un manuscrit secondaire, le Riccardianus 29 (vers 1495), adoptée par Musurus dans l'editio princeps de 1516 et à laquelle les éditeurs suivants sont restés inexplicablement fidèles. On obtient de la sorte un texte plus conforme à l'orthodoxie philologique et aussi un sens plus satisfaisant, car le respect de Pausanias à l'égard des interdits religieux rend peu vraisemblable qu'il ait divulgué un rite qui ne pouvait être connu « de tous ». En revanche, il a parfaitement pu dire ce qu'il estimait pouvoir dévoiler d'un rite qui

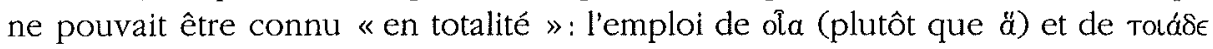

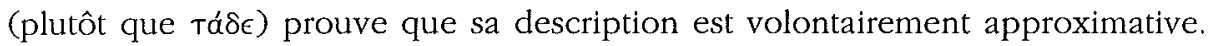


Autre divergence du texte proposé par rapport aux éditions courantes: au lieu

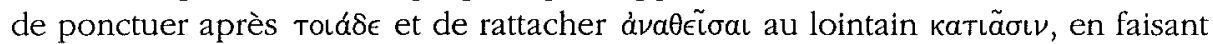
de $\epsilon^{\prime \prime} \mathrm{Tl} \delta \dot{\epsilon}$ - aủrouám une proposition incise explicative ${ }^{48}$, il me paraît plus

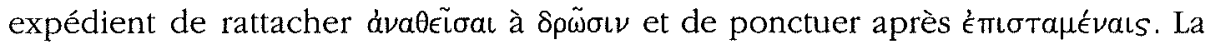
syntaxe s'en trouve allégée et la séquence topographique devient plus claire: après avoir évoqué ce qui se passe sur l'Acropole, le périégète nous transporte $\dot{\epsilon} v$ iñ $\pi 0 ́ \lambda \epsilon$, « en ville », où le rite s'achève.

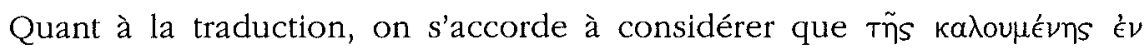

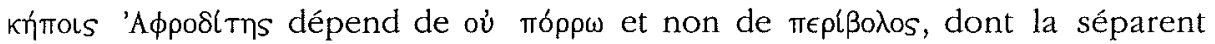

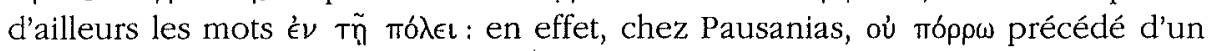
génitif commande toujours celui-ci ${ }^{49}$.

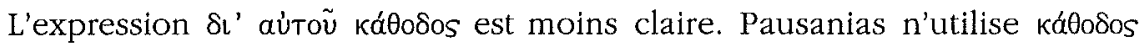
qu'à deux autres reprises pour désigner un passage matériel:

- dans le sanctuaire de Poséidon de l'Isthme de Corinthe, il signale un lieu

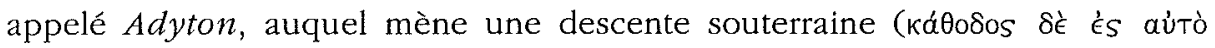
inó $\gamma \in \omega s)$;

- à Patras, devant le temple de Déméter, il mentionne une source à laquelle on

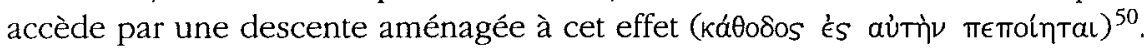

Dans les deux cas, le point d'aboutissement du passage est précédé de $\epsilon_{S}$. Pourquoi, dès lors, Pausanias emploie-t-il $\delta \iota^{\prime}$ aủTô̂ à propos de celui qu'empruntent les arrhéphores? Un seul parallèle peut être évoqué: parlant de l'enceinte sacrée ( $\epsilon \in$ pòs $\pi \epsilon \rho(\beta o \lambda o s)$ d'Apollon à Delphes, le périégète note que

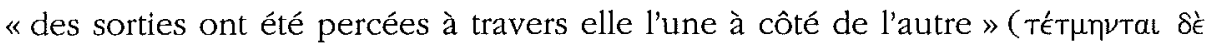

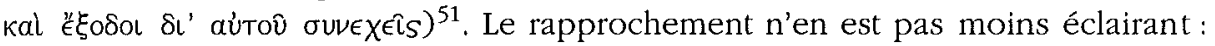

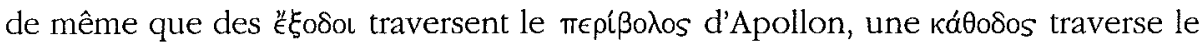
$\pi \epsilon \rho i \beta o \lambda o s$ d'Athènes, l'un et l'autre devant s'entendre au sens premier d'«enceinte $»$.

Passons aux problèmes d'interprétation, et d'abord à la localisation du

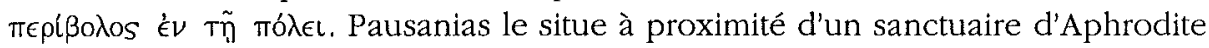
«dans les jardins ». Il a signalé auparavant un sanctuaire de ce nom entre

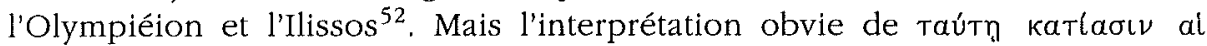

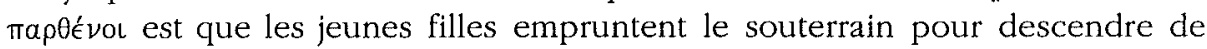
l'Acropole et être libérées « une fois en bas ». Or, un escalier aménagé dans une faille naturelle du rocher relie directement l'angle nord-ouest du plateau de la citadelle à une grotte située en contrebas. Immédiatement à l'est, le long du chemin de ceinture ( $є \epsilon \rho(\pi a т о \varsigma)$, un sanctuaire d'Aphrodite, désigné comme tel

\footnotetext{
48 À la suite de H. HITZıG et H. BLÜMNER éd., Pausaniae Graeciae descriptio, I, 1896, p. 295.

49 E. KADLETZ, $A J A, 86$ (1982), p. 445-446.

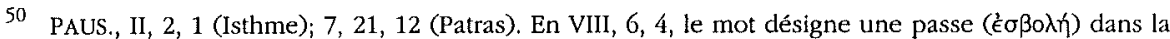
montagne.

51 PAUS., $X, 9,1$.

52 PAUS., I, 19, 2.
} 
par des dédicaces in situ, a été mis au jour en $1931^{53}$. Certes, les dédicaces ne qualifient pas explicitement la déesse d'Aphrodite «dans les jardins », mais la descente souterraine et l'enclos sacré se trouvent bien là où on les attendait et rien n'empêche Athènes d'avoir compté deux sanctuaires homonymes ${ }^{54}$.

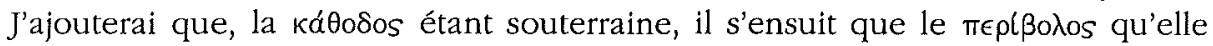
traverse est, au moins en partie, souterrain, lui aussi. La grotte répond à ce critère.

Elle a été longtemps considérée comme consacrée à Aglauros, dont le sanctuaire est désormais localisé avec certitude plus à l'est ${ }^{55}$. V. Pirenne-Delforge propose de l'attribuer à Hersé Courotrophos ${ }^{56}$. Je la suivrai d'autant plus volontiers que, selon Istros, les arrhéphores «(faisaient) la procession pour Hersé, fille de Cécrops » [L $\beta 2, \mathrm{~L} 3 \mathrm{a}]$. Il m'est, en effet, difficile de croire que cette information ait été forgée de toutes pièces pour justifier les formes en $\epsilon-$. En revanche, si la descente nocturne des arrhéphores aboutissait dans un sanctuaire d'Hersé, il ne fallait pas forcer beaucoup la réalité pour prétendre qu'elles faisaient la procession pour elle. Mais, m'objectera-t-on, cette descente peut-elle être assimilée à une processio n ? Un cortège pouvait parfaitement emprunter l'escalier, qui est large et dont la pente n'est pas particulièrement raide ${ }^{57}$, et Pausanias ne dit pas que les fillettes descendaient seules. Il est d'ailleurs invraisemblable qu'elles l'aient fait dans l'obscurité. Sauf si elles tenaient ellesmêmes une lampe en plus du fardeau qu'elles avaient sur la tête, elles devaient être accompagnées au moins de porteurs de torches. Et pourquoi pas de la prêtresse d'Athéna, dont on imagine mal qu'elle n'ait pas assisté à la relève des arrhéphores qui avait lieu « en bas »?

À propos de cette relève, la place de l'adverbe $\dot{\epsilon} \nu \tau \epsilon \tilde{u} \theta \epsilon \nu$ à la charnière des deux propositions de la dernière phrase, reliées par $\mu \dot{v} \nu$ - $\delta \dot{\epsilon}$, m'incite à le rapporter également au verbe äyovoıv et à considérer que les deux arrhéphores sortant de charge étaient remplacées sur-le-champ par deux autres, qui gagnaient aussitôt l'Acropole. Dans ce cas, on peut supposer qu'elles empruntaient pour s'y rendre - sous la conduite de la prêtresse d'Athéna? - le chemin

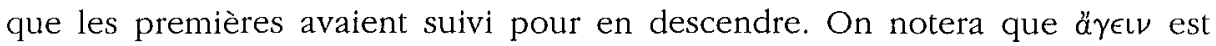
fréquemment employé au sens de «conduire en procession ». Nous n'avons, par contre, aucune raison de penser que les arrhéphores « libérées » remontaient sur l'Acropole ${ }^{58}$.

53 O. BRONEER, Hesperia, 1 (1932), p. 31-55; 2 (1933), p. 329-349; 4 (1935), p. 123-132; G.W. ELDERKIN, ibid, 10 (1941), p. 119-122.

54 Voir, en dernier lieu, PIRENne-Delforge, op. cit. (n. 3), p. 54-57. Nous ignorons pour lequel des deux sanctuaires Alcamène avait sculpté une statue célèbre (PLIN., Nat. bist., XXXVI, 16; LUCIAN., Imag, 4 et 6).

55 G.S. DONTAS, Hesperia, 52 (1983), p. 48-63.

56 PIRENNE-DELFORGE, op. cit. (n. 3), p. 57.

57 Cf. J. TRAvlos, Pictorial dictionary of ancient Atbens, Londres, 1971, p. 72 et 75, fig. 96 (reproduite dans PIRENNE-DELFORGE, op. cit. [n. 3], p. 58, fig. 5).

58 Comme le suppose PIRENNE-DELFORGE, op. cit. (n. 3), p. 53, n. 215. 


\section{Synthèse provisoire}

En combinant les diverses sources que j'ai sommairement passées en revue, on obtient de l'arrhéphorie une image, certes incomplète, mais relativement précise et cohérente. L'institution n'est pas attestée en dehors d'Athènes. Sans doute déjà ancienne au temps d'Aristophane [A1], elle s'est perpétuée au moins jusqu'au III $^{\mathrm{e}}$ siècle de notre ère, comme le prouvent les inscriptions [I24]. Nos sources évoquent surtout des arrhéphores au service d'Athéna Polias, qu'on peut considérer comme les arrhéphores «par excellence ». Mais cette divinité n'en avait pas l'exclusivité. Dès le $\mathrm{II}^{\mathrm{e}}$ siècle av. J.-C., sinon plus tôt, une ou plusieurs arrhéphores participent, au moins occasionnellement, aux Épidauries, c'est-à-dire au culte d'Asclépios [I8]. Une dédicace, que je suis tenté de dater de l'époque impériale romaine, pourrait évoquer une arrhéphore de Déméter et Coré [125]. Ce n'est peut-être pas un hasard si, à la même époque, une arrhéphore d'Athéna Polias est ensuite initiée du foyer et canéphore aux Éleusinies, deux fonctions en rapport avec la célébration des mystères d'Éleusis, puis canéphore aux Épidauries, ce qui nous ramène à Asclépios [I17]. En revanche, les (h)erséphores de Chloé Thémis et d'Ilithyie d'Agrai [I22-I23] sont sans doute des créations d'époque romaine à l'instar d'autres fonctions religieuses aux dénominations archaïsantes ${ }^{59}$.

Les arrhéphores se recrutaient dans l'aristocratie [L $\alpha$ ]. Les inscriptions confirment à cet égard le témoignage des lexiques: l'étude prosopographique des dédicaces de portraits d'arrhéphores tend, en effet, à montrer que leurs parents appartenaient à des familles en vue. Les sources littéraires vont dans le même sens. Les choreutes d'Aristophane ont - ou feignent d'avoir - été « élevée(s) brillamment dans le luxe » [A1]. Le client de Lysias [A2], qui a été triérarque, appartenait à la classe supérieure des contribuables athéniens; or, qui d'autres que les parents des arrhéphores pouvaient assurer le financement de l'arrhéphorie au titre de liturgie? Pisistrate était un aristocrate et le jeune Thrasybule faisait partie de la jeunesse dorée d'Athènes [A3]. Même la statue d'enfant à cheval qui se dressait dans le «jeu de balle » des arrhéphores avait un caractère aristocratique indéniable [A9]. On notera que la prêtresse d'Athéna Polias, qu'assistaient les arrhéphores «par excellence », et les ergastines, qui intervenaient aussi dans le tissage du voile de la déesse, appartenaient également à l'aristocratie.

Selon les lexiques, «quatre fillettes étaient élues [...] arrhéphores de sept jusqu'à onze ans » [La]. Ce mode de désignation rappelle celui des prêtresses, qui étaient également élues. Il ne valait pas pour toutes les arrhéphores: la fille de Léonidès, «donnée » par son père comme arrhéphore d'Asclépios [I8], n'a pas été élue. Il faut se rappeler ici que le culte de ce dieu n'a été introduit à Athènes qu'en 420 av. J.-C. par Sophocle et a eu d'abord un caractère privé. Nous

59 Cf. P. GRaINDOR, Athènes sous Hadrien, Le Caire, 1934, p. 140 sq.; G. DONNAY, BCH, 91 (1967), p. 546-551. 
ignorons quand et pourquoi on a inclu une ou des arrhéphore(s) dans la procession des Épidauries. Quant à l'âge des élues, la portée de l'expression ámò

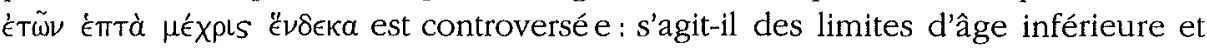
supérieure pour être admise à l'élection ou des âges du début et de la fin de la

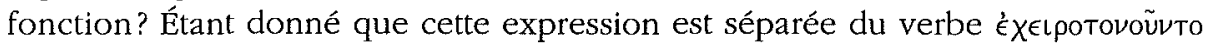

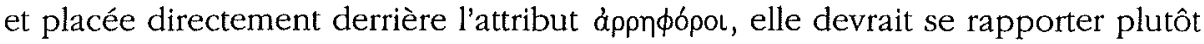
à la durée de la fonction: on devenait arrhéphore à sept ans et on cessait de l'être à onze. Que sept ans était l'âge minimum est confirmé par Aristophane [A1].

En toute hypothèse, les arrhéphores avaient moins de treize ans, qui était considéré dans l'Antiquité comme l'âge normal de la ménarché ${ }^{60}$. Cette limite d'âge doit être mise en rapport avec la pureté religieuse qui semble les avoir caractérisées [A7] et dont le port d'un vêtement blanc et de parures d'or, métal inaltérable, était sans doute le symbole [L6, L9a]. Élien [A10] les montre en compagnie de «femmes tout à fait pures »: ces dernières ne seraient-elles pas des femmes ménopausées qui, comme les fillettes impubères, échappaient à

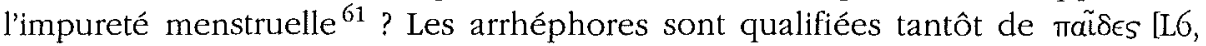

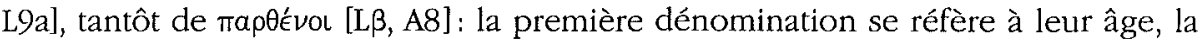
seconde à leur virginité. L'obligation de pureté explique sans doute la sévérité du châtiment que l'entourage de Pisistrate réclame pour le jeune Thrasybule, coupable d'avoir embrassé une arrhéphore. Elle se traduisait notamment par un régime alimentaire particulier $[\mathrm{A} 6, \mathrm{~L} 5 \mathrm{a}]^{62}$.

Parmi les quatre arrhéphores élues, deux étaient choisies pour accomplir différents rites relatifs au tissage du voile sacré d'Athéna [La]. Il doit s'agir des deux arrhéphores dont Pausanias [A8] signale la présence sur l'Acropole. Qui les

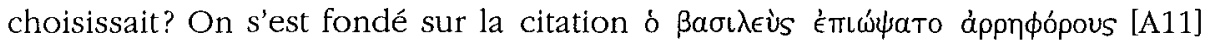
pour supposer que c'était l'archonte-roi, mais nous ignorons tout du contexte de cette citation et le roi qu'elle mentionne pourrait être un souverain mythique. Quel était le sort des deux autres élues? On estime généralement qu'elles ne devenaient pas arrhéphores. Toutefois, nos sources parlent de l'élection de quatre arrhéphores et non de l'élection de quatre fillettes parmi lesquelles on aurait choisi deux arrhéphores. Celles qui n'étaient pas occupées sur l'Acropole devaient l'être ailleurs, et pourquoi pas au service d'autres divinités? Dans ce cas, on pourrait songer à Déméter et Coré [I25].

Selon toute vraisemblance, les deux arrhéphores qui vivaient sur l'Acropole étaient les arrhéphores «par excellence », connues par les dédicaces de leurs portraits à Athéna Polias. Elles logeaient non loin du temple de la déesse qu'elles

60 BRULÉ, op. cit. (n. 1), p. 362.

61 Cf. BRULÉ, op. cit. (n. 1), p. 351 sq.

62 BURKerT, art. cit. (n. 3), p. 15, va cependant trop loin en affirmant que, dans le texte de Pausanias [A8], « das Wort $\delta$ (aıta [...] deutet auf bestimmte Tabus in ihrer Lebensführung 》 Slaıtav ËX 
servaient ${ }^{63}$. Nous ne sommes guère renseignés sur la vie qu'elles y menaient: le « jeu de balle » [A9] servait-il à la détente des fillettes ou avait-il une fonction rituelle $^{64}$ ? Parmi les rites auxquels elles participaient, nos sources mentionnent la pose de la trame du voile sacré d'Athéna, qui avait lieu le dernier jour de pyanepsion, jour de la fête des Chalkéia [L4b, L5f]. Pausanias [A8] décrit le rituel de leur sortie de charge, dont les archéologues pensent avoir retrouvé le décor. Elles descendaient de nuit, par un escalier souterrain, jusqu'à une grotte - peut être consacrée à Hersé - en portant sur la tête des objets secrets, contenus dans des récipients fermés puisque ni la prêtresse d'Athéna, qui les leur avait remis, ni elles-mêmes n'en connaissaient la nature. Une fois en bas, elles déposaient leur farcleau, recevaient en échange «un autre objet qu'elles emport(ai)ent enveloppé » et, peut-on croire, rentraient chez elles, tandis que deux nouvelles arrhéphores prenaient leur place sur l'Acropole.

J'ai qualifié plus haut ce rite nocturne de procession. Une des fonctions des arrhéphores était précisément de participer à des processions [L/2, L3a, A5]. L'élément final (-фópos) de leur nom se rapporte d'ailleurs, selon toute vraisemblance, à cette fonction, même si l'interprétation de l'élément initial

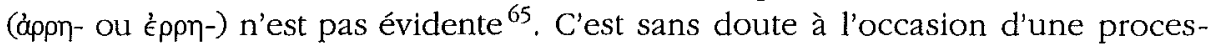
sion que Thrasybule a aperçu la fille de Pisistrate áppnфopoũoav [A3]. C'est sans doute pour participer à la procession des Épidauries que Léonidès a donné sa

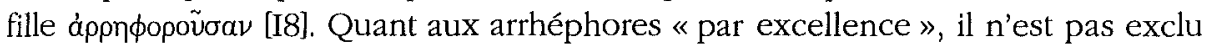
qu'elles aient aussi joué un rôle dans la procession des Panathénées : sur la frise du Parthénon, on voit deux fillettes intervenir dans ce qui semble être la remise du voile sacré à un magistrat (?).

Les lexiques définissent le mot áppnфopía comme désignant une fête [L $\beta_{1}$ ]. On a mis en doute que ce mot accentué paroxyton, donc au féminin singulier, puisse avoir ce sens, les noms grecs des fêtes étant normalement au neutre pluriel, Mais, en scrutant les apparats critiques des lexiques, on découvre çà et là

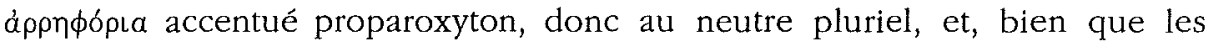
éditeurs n'aient pas retenu ces leçons minoritaires, la possibilité existe d'une

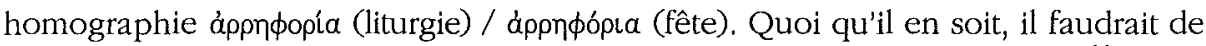
plus solides raisons pour récuser le témoignage unanime des lexiques ${ }^{66}$, dont nous avons vu qu'il provient, en général, de sources fiables.

63 G.P. STEVENS, Hesperia, 5 (1936), p. 486-491, identifiait leur demeure avec un bâtiment dont les vestiges ont été mis au jour au nord-ouest de l'Érechthéion, mais cette identification a été contestée par K. JEPPESEN, AJA, 83 (1979), p. 381-394.

64 Sic BURKERT, art. cit. (n. 3), p. 11.

65 Cf. VAN SICHELEN, art. cit. (n. 1), p. 98-99; ROBERTSON, art. cil. (n. 3), p. 244 sq. Je laisse volontairement de côté l'épineuse question de la véritable étymologie d'áppnфópos.

66 Pour L9b, voir ci-dessous n. 68. 
Cette fête était « célébrée en l'honneur d'Athéna au mois de scirophorion $^{67} »$. Il est tentant de l'identifier à celle au cours de laquelle s'effectuait la relève des deux arrhéphores de l'Acropole, bien que Pausanias [A8] n'en donne ni le nom ni la date. En effet, les lexicographes précisent que, lors de la fête qu'ils

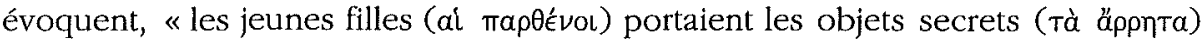
dans des cistes » $\left[\mathrm{L} \beta_{2}\right]$. On notera les deux articles définis : il s'agit de jeunes filles déterminées, qui ont toute chance d'être les arrhéphores "par excellence », portant des objets déterminés, dont les Athéniens savaient qu'ils étaient contenus dans les cistes, tout en ignorant leur nature. Peut-on identifier également la fête en question aux Arrhétophories de Clément d'Alexandrie [A12] et du scholiaste de Lucien [S2]? Ce que ce dernier décrit de la célébration des Thesmophories semble bien se rapporter à la fête athénienne de ce nom. Étant donné que les Scirophories ne sont pas attestées en dehors d'Athènes, on peut supposer que la totalité de la scholie se réfère à des réalités religieuses de cette cité. Dès lors, les Arrhétophories dont elle parle seraient une fête athénienne, qui pourrait difficilement ne pas être la même que sa quasi homonyme des lexiques. Dans ce cas, les objets secrets que portaient les arrhéphores seraient des phallus et des serpents «façonnés en pâte à pain » et l'objet qu'elles recevaient en échange, une branche de pin ${ }^{68}$.

\section{Rite d'initiation ou rite civique ? Essai de conclusion}

Une revue critique des exégèses de l'arrhéphorie qui ont été proposées à ce jour dépasserait le cadre d'un article. On peut néanmoins les répartir sommairement en deux catégories: celles qui y voient une initiation et les autres. Les premières ne tiennent compte, en fait, que des deux arrhéphores " par excellence » et, surtout, du rite nocturne qui marquait leur sortie de charge. Elles négligent les autres, dont nous ne savons, il est vrai, pas grand-chose. Quels arguments invoquent-elles?

1. L'exclusion temporaire de la communauté: Certes, les deux fillettes séjournaient un certain temps sur l'Acropole, mais elles ne semblent nullement y avoir été recluses. Au contraire, elles participaient à divers rites collectifs et rien ne dit qu'elles ne recevaient pas de visites.

67 BURKERT, art. cit. (n. 3), p. 5, n. 2, met en rapport avec l'arrhéphorie une série de sacrifices mentionnés à la date du 3 scirophorion dans le calendrier sacrificiel du dème d'Erchia (G. DAUX, $B C H, 87$ [1963], p. 606-610, col. A, 57-65; B, 55-59; $\Gamma, 59-64, \Delta ; 56-60)$. Il refuse toutefois d'en déduire que la fête athénienne avait lieu à la même date - ce qui ne l'empêchera pas d'être généralement adoptée dans les travaux ultérieurs. Contra: ROBERTSON, art. cit. (n. 3), p. 281-282.

68 Est-ce à cause de cette branche de pin qu'un lexique [L9b] définit l'arrhéphorie comme une

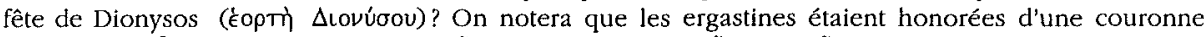

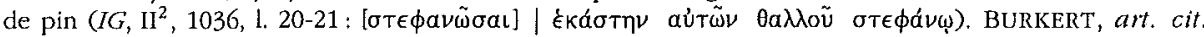
(n. 3), p. 18, tire argument du participe $\epsilon \gamma \kappa \epsilon \kappa a \lambda u \mu \mu \epsilon \nu o \nu$ pour imaginer que les arrhéphores descendant de charge emportaient un (simulacre de) bébé. Pour RoBERTSON, art. cit. (n. 3), p. 265276 , il s'agissait plutôt d'une pierre. 
2. L'initiation aux travaux féminins: Si les deux fillettes intervenaient dans le tissage du voile sacré d'Athéna, on ignore en quoi consistait cette intervention. On sait seulement qu'elles assistaient les prêtresses lors de la pose de la trame. Par contre, le tissage proprement dit était effectué par des jeunes filles plus âgées, les ergastines ${ }^{69}$.

3. L'initiation à la sexualité: Nul ne prétend plus que le sanctuaire d'Aphrodite mentionné par Pausanias soit autre chose qu'un simple repère topographique. Il est donc entendu que cette déesse ne jouait aucun rôle dans le rituel arrhéphorique. Mais chassez Aphrodite, elle revient au galop, et l'on nous invite à relire le témoignage du périégète à la lumière d'un parallèle italien. $\grave{A}$ Lanuvium, une fois par an, des jeunes filles effrayées descendent nuitamment dans une grotte, les yeux bandés, pour porter des gâteaux d'orge à un serpent affamé; quand elles remontent, les paysans s'écrien $t:$ «'année sera fertile » ${ }^{70}$. Il faut beaucoup de bonne volonté pour comparer les deux rites: on ne bande pas les yeux des arrhéphores; rien, dans le texte de Pausanias, n'indique qu'elles aient été effrayées, ni qu'on ait voulu les effrayer; elles ne portent pas de la nourriture, mais des arrbèta, il n'y a pas de serpent dans la grotte où elles se rendent... Je ne suis d'ailleurs pas convaincu que le rite de Lanuvium ait été une « ordalie virginale ${ }^{71}$. S'il fallait absolument lui trouver un parallèle grec, je songerais plutôt aux Thesmophories ${ }^{72}$ et à la descente des "puiseuses » dans les megara, que hantaient aussi des serpents voraces, d'autant que les deux rites avaient pour but d'assurer une bonne récolte. En outre, l'âge tendre des arrhéphores et la pureté qu'elles sont tenues de préserver excluent, à mon sens, toute référence à la sexualité. Elles sont, d'autre part, trop peu nombreuses pour représenter une classe d'âge.

Si l'arrhéphorie n'est pas une initiation, alors qu'est-ce? La question reste posée, mais un certain nombre de pistes d'interprétation se dégagent, à mon sens, de l'ensemble des sources. Tout d'abord, l'image qu'elles nous donnent de l'arrhéphorie n'est pas celle d'un rite ponctuel, mais plutôt d'un sacerdoce qui, pour les quatre arrhéphores élues, durait sans doute pas moins de quatre années. Le nom de ce sacerdoce se réfère à une fonction : porter des objets lors de processions. La pureté exigée des arrhéphores peut d'ailleurs être mise en rapport avec la sacralité particulièrement forte des objets secrets qu'on leur confiait. Mais leur rôle ne se limitait pas à cela. Celles qui vivaient sur l'Acropole assistaient notamment la prêtresse d'Athéna Polias dans l'accomplissement de divers rites en relation avec le tissage du voile sacré qu'on offrait à la déesse lors

\footnotetext{
69 BRULE, op. cit. (n. 1), p. 100 sq.

70 Propert., IV, 8, 3-16. Cf. AELIAN., Nat. anim., XI, 16; PYTHOCLES, FGrHist, 833 F 1 a.

71 BRUlé, op. cit. (n. 1), p. 95 sq., suivi par PIRENNE-DELFORGE, op. cit. (n. 3), p. 54 . La comparaison des deux rites est empruntée à ROBERTSON, art. cit. (n. 3), p. 258 sq., qui les considère, lui, comme des rites propitiatoires.

72 Ci-dessus n. 37.
} 
des Grandes Panathénées; il est regrettable que nos sources soient muettes sur ce que faisaient les autres.

Quant au fameux rituel nocturne, je l'interprète, non comme une épreuve initiatique, mais comme une procession au cours de laquelle les arrhéphores sortant de charge transportaient les arrbèta hors de l'Acropole et les remettaient à leurs remplaçantes, qui les y ramenaient. Elle a lieu de nuit pour que la substitution s'opère à l'abri des regards divins; ainsi se perpétue la présence de deux fillettes d'âge convenable dans le sanctuaire d'Athéna. Il est probable que cette présence permanente était ressentie comme indispensable à la survie, voire à la prospérité de la Cité. Servantes de la déesse poliade, vivant auprès d'elle sur l'Acropole, gardiennes de mystérieux arrbèta, recrutées dans l'aristocratie, les arrhéphores «par excellence » semblent bien relever d'une strate ancienne de la religion athénienne. Les liens, relevés par Jane Emily Harrison dès 1889, entre le rituel arrhéphorique et le mythe des Cécropides nous orientent vers le passé royal d'Athènes; une intervention de l'archonte-roi dans le choix des fillettes, même si elle n'est pas prouvée, ne serait donc pas incongrue. La relève des arrhéphores a lieu en scirophorion, qui est le dernier mois du calendrier attique: ne pourrait-elle s'inscrire parmi les rites qui marquaient le renouvellement de l'année? Dans cette perspective, il vaudrait la peine d'approfondir ses relations avec les Panathénées ${ }^{73}$, mais aussi peut-être avec les Scirophories.

Il reste encore beaucoup de signes à décoder avant d'aboutir à une interprétation satisfaisante, si cela s'avère possible. Puisse ce modeste article y avoir contribué en dégrossissant les matériaux et en balisant le terrain.

Université Libre de Bruxelles

GuY DONNAY

Avenue F.D. Roosevelt, 50 - CP 175

B - 1050 BRUXELLES

73 Dans lesquelles BURKERT, art. cit. (n. 3), p. 22 sq., reconnaît un rite royal de renouvellement de l'année. 


\section{Liste des sources écrites}

Dans chaque catégorie, les témoignages ont été classés, autant que possible, par ordre chronologique.

\section{Auteurs}

A1 ARISTOPHANE, Lysistrata, 641-642

A2 LYSIAS, Discours, 21, 5

A3 ANONYME, Dialogue sur le pouvoir $=$ POxy 664, 1. 29-32

A4 MÉNANDRE, fr. 59-67 Körte et fr. 104 Austin

- Dinarque, Contre Pytbéas, fr. 6, 4 Conomis = L1

- Callimaque, fr. 520 Pfeiffer $=$ L4b, L5f

- CAllimaQue, fr. 741 Pfeiffer $=$ L4a

- ISTROS, FGrHist $334 \mathrm{~F} 27=\mathrm{S1}$

A5 DENYS D'HALICARNASSE, Antiquités romaines, II, 22, 2

A6 TrYPhON, fr. 116 von Velsen = ATHÉNÉE, Deipnosopbistes, III, $114 \mathrm{a}$

A7 PhILON D'ALEXANDRIE, De l'ébriété, 129

A8 PAUSANIAS, I, 27, 3

A9 Ps.-PLUTARQUE, Vies des dix orateurs, p. $839 \mathrm{C}$

A10 ÉlIEN, fr. 310 Herscher

A11 ANONYME (ÉliEN?) [PLATON le Comique, fr. $263 \mathrm{Kock}$

A12 ClÉment D'AleXANDRIE, Protreptique, II, 17, 1
411 av. J.-C. début du $\mathrm{IV}^{\mathrm{e}}$ s. av. J.cC. $\mathrm{IV}^{\mathrm{e}} / \mathrm{III}^{\mathrm{e}}$ s. av. J.-C. $\mathrm{IV}^{\mathrm{e}} / \mathrm{III}^{\mathrm{e}}$ s, av, J,-C. $\mathrm{IV}^{\mathrm{e}} / \mathrm{III}^{\mathrm{e}}$ s, av, J.-C. III $^{e}$ s. av. J.-C. III $^{\mathrm{e}}$ s. av. J.-C. $I^{\text {er }}$ s. av. J.-C. $\mathrm{I}^{\text {er }}$ s. av, J.-C. $\mathrm{I}^{\mathrm{er}}$ s. av. J.-C./ $/ \mathrm{I}^{\mathrm{er}}$ s. ap. J.-C. II $^{\mathrm{e}}$ s. ap. J.-C. $\mathrm{II}^{\mathrm{e}}$ s. ap. J.-C. ou après $\mathrm{II}^{\mathrm{e}} / \mathrm{III}^{\mathrm{e}}$ s. ap. J.-C. $\mathrm{Il}^{\mathrm{e}} / \mathrm{III}^{\mathrm{e}}$ s. ap. J.-C. (?) $\mathrm{II}^{\mathrm{e}} / \mathrm{III}^{\mathrm{e}}$ s. ap. J.-C.

\section{Lexiques}

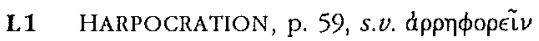

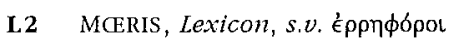

$\mathrm{I}^{\mathrm{er}} / \mathrm{II}^{\mathrm{e}}$ s. ap. J.-C. (?)

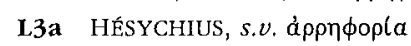

II $^{\mathbf{e}}$ s. ap. J.-C,

$\mathrm{V}^{\mathrm{e}}$ s. ap. J.-C.

L3b ID., s.v. ả $\rho \rho \eta \phi o ́ p o s$

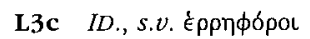

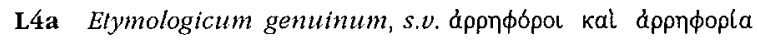

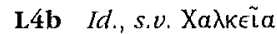

L5a Souda, s.v. áváotatol

IXe s. ap. J.-C.

$\mathrm{X}^{\mathrm{e}}$ s. ap. J.-C.

L.5b Id., s. $v$. à $\rho p \eta \nu \circ о \rho \in i ̈ \nu$

L5c Id., s.v. áppnфopía

L5d Id., s.v. É $\rho \sigma \varepsilon \phi o \rho l \alpha$

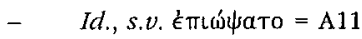

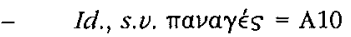

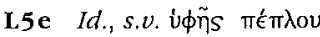

L5f $I d, s, v$. Хa $\alpha \kappa \varepsilon \tilde{i} \alpha$

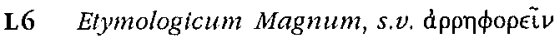

XII $^{\mathrm{e}}$ s. ap. J.-C.

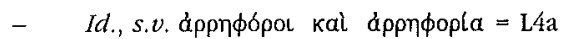

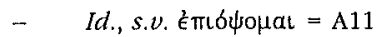

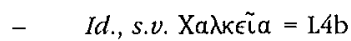

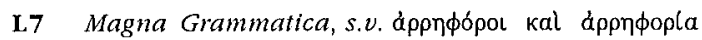

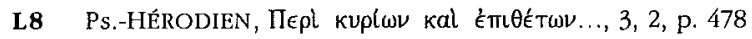

date inconnue

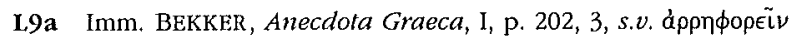

date inconnue 


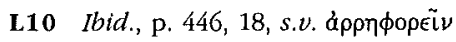

date inconnue

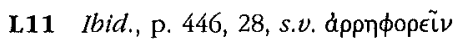

date inconnue

\section{Scholies}

S1 Scholie à ARISTOPHANE, Lysistrata, 642

s2 Scholie à LUCIEN, Dialogtue des coturtisanes, 2, 1

\section{Inscriptions}

I1 Dédicace (à Athéna?) d'une statue d'errhéphore sous la prêtrise de Call[---] et l'archontat de Ménécratès $\left(I G, \mathrm{II}^{2}, 3461\right)$

220/19 av. J.-C.

I2 Dédicace à Athéna d'une statue de l'errhéphore (?) [--s]istraté par son père [Hilppoclès de Sypallétos et sa mère [- -llyra $\left(I G, \mathrm{I}^{2}, 3465\right)$

13 Dédicace (à Athéna) de la statue d'une errhéphore sous la prêtrise (?) de [--]médéia et l'archontat(?) de [--]codr[--] $\left(I G, \mathrm{II}^{2}, 3466\right)$

I4 Dédicace à Athéna d'une statue de l'errhéphore Anthémia par son père [Apo]llon[ios] d'Aphidna, son oncle Oulialdès] et sa mère Philot[éra] sous la prêtrise de Pentétéris fille d'Hiéroclès de Phlyées, œuvre de Caicosthénès et Diès $\left(I G, \mathrm{II}^{2}, 3470\right)$

I5 Dédicace à Athéna d'une statue de l'errhéphore [---]sté par son père Ant[---] sous la prêtrise de Pentétéris fille d'Hiéroclès de Phlyées $\left(I G, \mathrm{II}^{2}, 3471\right)$

I6 Dédicace à Athéna et Pandrose d'une statue de l'errhéphore Phila par son père [--]os fils de Dionysiclès de Trinéméa sous la prêtrise de Théodoté fille de Polyoctos d'Amphitropé, œuvre de Caicosthénès (IG, $\mathrm{II}^{2}, 3472$ )

200-150 av. J.-C.

17 Dédicace à Athéna d'une statue de l'errhéphore Xénostraté par son père Agias fils de Nicarchos d'Euonymée, sa mère Démétria fille de Boulon de Péania et ses frères Arcétos, Épicratès, Boulon et Xénophon sous la prêtrise de Phi[l]o[téra] $\left(I G, \mathrm{Il}^{2}, 3473\right)$

c. 150 av. J.-C.

18 Décret en l'honneur de Léonidès fils de Nicocratès, ancien prêtre d'Asclépios à Athènes, qui a notamment « donné sa propre fille [.... lors des] Épidauries en qualité d'arrhéphore $\gg\left(I G, \mathrm{II}^{2}, 974 ;\right.$ O. HUBBLE, Hesperia, 28, 1959, p. 188-194, $\mathrm{n}^{\circ}$ 10; S.B. AlESHIRE, Asklepios at Athens, Amsterdam, 1991, p. 91-92)

$137 / 6$ av. J.-C.

19 Dédicace à Athéna d'une statue de l'errhéphore Pha[---] $\left(I G, I^{2}, 3486\right) \quad \mathrm{II}^{\mathrm{e}} \mathrm{s}$. av. J.C.

I10 Dédicace à Athéna Polias d'une statue de l'errhéphore Polycratéia par son père (nom perdu) et sa mère [---] fille d'Antaménès de Mélité sous la prêtrise de Chrysis fille de Nicétos de Pergasé $\left(I G, \mathrm{II}^{2}, 3482\right)$

fin du $\mathrm{II}^{\mathrm{e}} \mathrm{s}$. av. J.-C.

111 Dédicace à Athéna Polias [et Pandrose] d'une statue de l'errhéphore Panarista par son père Mantias de Marathon, sa mère Théodoté fille de Dosithéos de Myrrhinonte et ses frères Cléoménès et $[--]\left(I G, \mathrm{Il}^{2}, 3488\right) \quad$ début du $\mathrm{I}^{\mathrm{er}} \mathrm{s}$. av. J.-C.

112 Dédicace à Athéna [Polias] d'une statue de l'errhéphore Athén[---] fille de Théodoros de Marathon par son grand-père Hipponlikos] $\left(I G, \mathrm{II}^{2}, 3496\right) \quad \mathrm{I}^{\mathrm{er}} \mathrm{s}$. av. J.-C.

113 Dédicace à Athéna d'une statue de l'errhéphore Stratonice par son père [Pleisti]as fils d'Aichmaios de Céphisia, sa mère Pythias fille de Diodoros d'Amphitropé et ses frères Diodoros et Pleistias sous la prêtrise de Stratocléia $\left(I G, \mathrm{II}^{2}, 3497\right)$

114 Dédicace à Athéna Polias et Pandrose d'une statue de l'errhéphore Nausicraté par son père [---]on d'Azénia et sa mère [- - - ] fille de [---]on de Lamptrai sous la prêtrise de Callist---] $\left(I G, \mathrm{II}^{2}, 3515\right)$

époque d'Auguste

I15 Dédicace d'une statue d'une inconnue qui a été errhéphore pour Athéna [Polias] sous la prêtrise d'Alexandra fille de léon de Cholléides $\left(I G, \mathrm{II}^{2}, 3516\right)$

époque d'Auguste

I16 Dédicace d'une statue de l'arrhéphore Athénaïs par son père Patricos et sa mère Eucléia $\left(I G, \mathrm{II}^{2}, 3528\right)$ début du I ${ }^{\text {er }}$ s. ap. J.-C. 
$\mathbf{1 7}$ Dédicace par le Conseil et le Peuple d'une statue de Tertia fille de Lucius [---] qui a été errhéphore pour Athéna Polias sous la prêtrise de Stratocléia $\left(I G, \mathbf{I I}^{2}\right.$, 3554)

$$
\text { 'er s. ap. J.C. }
$$

118 Dédicace par le Conseil et le Peuple d'une statue d'Apollodora fille d'Apollodloros] de Gargèttos qui a été errhéphore pour Athéna Polias $\left(I G, \mathrm{II}^{2}, 3556\right) \mathrm{I}^{\mathrm{er}} \mathrm{s}$. ap. J.-C.

I19 Dédicace d'une statue de [---] fille de [---]ios d'CEon, qui a été errhéphore pour Athéna Polias, par sa mère Hélène fille d'A[---] de Marathon sous la prêtrise de $[--]\left(I G, \mathrm{II}^{2}, 3555\right)$

I20 Dédicace sur décret du Conseil des Cinq Cents d'un hermès-portrait de Glaucos fils de Memnon d'Anaphlyse par sa mère, l'ex-arrhéphore Télété fille de Glaucos de Céphisia, à la requête de son mari Claudius Atticus (IG, II' ${ }^{2}$,3960)après 128/129 ap. J.-C.

I21 Dédicace métrique à une déesse non nommée (Athéna?) d'une statue de l'errhéphore Théano par son père Sarapion, sa mère [Ch]ré[simé] et cinq frères $\left(I G, \mathrm{II}^{2}, 3634\right)$

après 150 ap. J.-C.

I22 Inscription réservant des sièges du théâtre de Dionysos aux deux (h)erséphores de Chloé Thémis $\left(I G, \mathrm{I}^{2}, 5098\right)$

123 Inscription réservant des sièges du théâtre de Dionysos aux deux (h)erséphores d'llithyie à Agrai $\left(I G, \mathrm{II}^{2}, 5099\right)$

124 Dédicace de deux statues en action de grâce à Amphiaraos par les ex-spondophores Aurélius Calliphron et Aurélius Patrocle fils de l'ex-archonte éponyme Aurélius Calliphron et de ''(ex-) arrhéphore Claudia Antonina (J.H. OLIVER, AJA 1941, p. 541-542)

125 Dédicace à Déméter et Coré d'une statue d'une errhéphore fille ou petite-fille d'Aristoclès $\left(I G, \mathrm{II}^{2}, 3729\right)$ 